\title{
Level of Consciousness Is Dissociable from Electroencephalographic Measures of Cortical Connectivity, Slow Oscillations, and Complexity
}

\author{
DDinesh Pal, ${ }^{1,2,3 *}$ Duan Li, ${ }^{1,3 *}$ Jon G. Dean, ${ }^{3,4}$ Michael A. Brito, ${ }^{2,3}$ Tiecheng Liu, ${ }^{1}$ Anna M. Fryzel, ${ }^{1}$ Anthony G. Hudetz, ${ }^{1,2,3}$ \\ and George A. Mashour ${ }^{1,2,3}$ \\ ${ }^{1}$ Department of Anesthesiology, University of Michigan, Ann Arbor, Michigan 48109-5615, ${ }^{2}$ Neuroscience Graduate Program, University of Michigan, Ann \\ Arbor, Michigan 48109-2215, ${ }^{3}$ Center for Consciousness Science, University of Michigan, Ann Arbor, Michigan 48109, and ${ }^{4}$ Department of Molecular and \\ Integrative Physiology, University of Michigan, Ann Arbor, Michigan 48109-5622
}

Leading neuroscientific theories posit a central role for the functional integration of cortical areas in conscious states. Considerable evidence supporting this hypothesis is based on network changes during anesthesia, but it is unclear whether these changes represent state-related (conscious vs unconscious) or drug-related (anesthetic vs no anesthetic) effects. We recently demonstrated that carbachol delivery to prefrontal cortex (PFC) restored wakefulness despite continuous administration of the general anesthetic sevoflurane. By contrast, carbachol delivery to parietal cortex, or noradrenaline delivery to either prefrontal or parietal cortices, failed to restore wakefulness. Thus, carbachol-induced reversal of sevoflurane anesthesia represents a unique state that combines wakefulness with clinically relevant anesthetic concentrations in the brain. To differentiate the state-related and drug-related associations of cortical connectivity and dynamics, we analyzed the electroencephalographic data gathered from adult male Sprague Dawley rats during the aforementioned experiments for changes in functional cortical gamma connectivity $(25-155 \mathrm{~Hz})$, slow oscillations $(0.5-1 \mathrm{~Hz})$, and complexity $(<175 \mathrm{~Hz})$. We show that higher gamma $(85-155 \mathrm{~Hz})$ connectivity is decreased $(p \leq 0.02)$ during sevoflurane anesthesia, an expected finding, but was not restored during wakefulness induced by carbachol delivery to PFC. Conversely, for rats in which wakefulness was not restored, the functional gamma connectivity remained reduced, but there was a significant decrease $(p<0.001)$ in the power of slow oscillations and increase $(p<0.001)$ in cortical complexity, which was similar to that observed during wakefulness induced after carbachol delivery to PFC. We conclude that the level of consciousness can be dissociated from cortical connectivity, oscillations, and dynamics.

Key words: anesthesia; cholinergic; consciousness; EEG; functional connectivity; noradrenergic

Significance Statement

Numerous theories of consciousness suggest that functional connectivity across the cortex is characteristic of the conscious state and is reduced during anesthesia. However, it is unknown whether the observed changes are state-related (conscious vs unconscious) or drug-related (drug vs no drug). We used a novel rat model in which cholinergic stimulation of PFC produced wakefulness despite continuous exposure to a general anesthetic. We demonstrate that, as expected, general anesthesia reduces connectivity. Surprisingly, the connectivity remains suppressed despite pharmacologically induced wakefulness in the presence of anesthetic, with restoration occurring only after the anesthetic is discontinued. Thus, whether an animal exhibits wakefulness or not can be dissociated from cortical connectivity, prompting a reevaluation of the role of connectivity in level of consciousness.

\section{Introduction}

The biological basis of consciousness is considered to be among the most fundamental questions in science. A number of prominent theories of consciousness focus on the integration of neural

Received Aug. 6, 2019; revised Nov. 5, 2019; accepted Nov. 22, 2019

Author contributions: D.P. and G.A.M. designed research; D.P., J.G.D., M.A.B., T.L., and A.M.F. performed research; D.P., D.L., J.G.D., and M.A.B. analyzed data; D.P. wrote the first draft of the paper; D.P., D.L., J.G.D., M.A.B., A.G.H., and G.A.M. edited the paper; D.P., D.L., and G.A.M. wrote the paper; A.G.H. contributed unpublished reagents/analytic tools. information in cortical networks as measured via the strength or repertoire of functional brain connections (Dehaene and Changeux, 2011; Baars et al., 2013; Tononi et al., 2016; Carhart-Harris, 2018). Studies across multiple species and from multiple labora-
This work was supported by National Institutes of Health Grant R01 GM111293 to G.A.M. and D.P., and funding from the Department of Anesthesiology, University of Michigan Medical School, Ann Arbor, Michigan. We thank Dr. Chris Andrews (Consulting for Statistics, Computing \& Analytics Research, University of Michigan, Ann Arbor, Michigan) for help with statistical analysis.

The authors declare no competing financial interests. 
tories, including our own, suggest that (1) disruption of frontal-parietal functional connectivity (Lee et al., 2009, 2013; Ku et al., 2011; Schrouff et al., 2011; Boly et al., 2012; Hudetz, 2012; Jordan et al., 2013; Bonhomme et al., 2016; Pal et al., 2016; Ranft et al., 2016; Schroeder et al., 2016; Li et al., 2017; Bodart et al., 2018; Sanders et al., 2018a), (2) increase in spectral power of slow oscillations (Lewis et al., 2012; Ní Mhuircheartaigh et al., 2013; Purdon et al., 2013; Warnaby et al., 2017), and (3) reduced spatiotemporal complexity (Casali et al., 2013; Hudetz et al., 2015,

2016; Sarasso et al., 2015; Schartner et al., 2015; Li and Mashour, 2019) are correlates of anesthetic-induced unconsciousness. However, it is not clear whether the anesthetic-induced disruption in cortical connectivity, increase in the spectral power of slow oscillations, and reduction in cortical complexity correlate with unconsciousness (i.e., a state effect) or reflect the presence of anesthetic drugs in the brain (i.e., a drug effect). A primary reason for this gap in our understanding is that, normally, the presence of anesthetic drugs in the brain at clinically relevant concentrations is associated with the absence of wakefulness. Conversely, wakefulness normally occurs in the absence of anesthetic drugs or when these drugs reach subanesthetic concentrations in the brain. In a recent study (Pal et al., 2018), we demonstrated that cholinergic stimulation, via local carbachol delivery, of prefrontal cortex (PFC) in sevoflurane-anesthetized rat was sufficient to restore wakefulness despite the continuous presence of sevoflurane at concentrations $(1.9 \%-2.4 \%)$ associated with surgical anesthesia. If preserved frontal-parietal connectivity, low power of slow oscillations, and/or high spatiotemporal complexity are indeed correlates of wakefulness, then these should be restored during carbachol-induced wakefulness despite the presence of anesthetic in the brain. Conversely, if disruption of frontal-parietal connectivity, enhanced power of slow oscillations, and/or reduced spatiotemporal complexity correlate with the presence of anesthetics in the brain, then pharmacological restoration of wakefulness in the presence of sevoflurane will not be associated with restoration to baseline wake levels. Interestingly, in our previous study (Pal et al., 2018), we also demonstrated that cholinergic stimulation of parietal cortex or the noradrenergic stimulation of either prefrontal or parietal cortices in sevofluraneanesthetized rats activated the electroencephalogram (EEG) but failed to reverse general anesthesia and restore wakefulness, creating alternative models for understanding the state- and drug-related effects of general anesthetics.

In the current study, we analyzed the EEG datasets collected as part of these experiments (Pal et al., 2018) and demonstrate that (1) functional cortical gamma connectivity remains suppressed during ongoing sevoflurane exposure despite the concurrent induction of wakefulness following carbachol delivery into PFC; and (2) the changes in slow oscillations and cortical complexity correlate with changes in EEG activation rather than behavior. These findings suggest that the level of consciousness can be dissociated from EEG measures of connectivity and dynamics,

*D.P. and D.L. contributed equally to this work.

Correspondence should be addressed to Dinesh Pal at dineshp@med.umich.edu.

https://doi.org/10.1523/JNEUROSCI.1910-19.2019

Copyright @ 2020 Pal, Li et al.

This is an open-access article distributed under the terms of the Creative Commons Attribution License Creative Commons Attribution 4.0 International, which permits unrestricted use, distribution and reproduction in any medium provided that the original work is properly attributed.
Continuous microdialysis sample collection and electroencephalographic recording

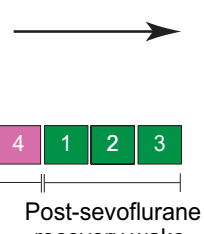

recovery wake

Figure 1. Schematic illustrating the experimental design. The EEG and microdialysis data were gathered simultaneously and without any interruption, but the dialysis samples were collected in $12.5 \mathrm{~min}$ bins, as illustrated by individual boxes in the

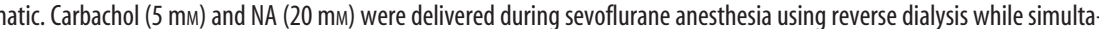
neously collecting the dialysis fractions for quantification of the local acetylcholine levels (Pal et al., 2018). The same experimental

prompting a reevaluation of the precise role in the mechanism and monitoring of consciousness.

\section{Materials and Methods}

We used the intracranial EEG data from our recently published study (Pal et al., 2018) to analyze the changes in functional cortical gamma connectivity $(25-155 \mathrm{~Hz})$, spectral power of slow oscillations $(0.5-1 \mathrm{~Hz})$, and Lempel-Ziv complexity $(<175 \mathrm{~Hz})$, before, during, and after reverse dialysis delivery of carbachol and noradrenaline (NA) into the PFC and parietal cortex of sevoflurane-anesthetized rats. All experiments were conducted in adult male Sprague Dawley rats (300-350 g, Charles River Laboratories) maintained on a $12 \mathrm{~h}$ light $/ 12 \mathrm{~h}$ dark cycle (lights on at 6:00 A.M.) with ad libitum food and water. The experiments were approved by the Institutional Animal Care and Use Committee (University of Michigan, Ann Arbor, Michigan) and were in compliance with the Guide for the care and use of laboratory animals (Ed 8, National Academies Press) as well as the ARRIVE guidelines. The EEG data were collected from five cortical sites: one frontal (from bregma: anterior $3.0 \mathrm{~mm}$ and mediolateral $2.5 \mathrm{~mm}$ ), two parietal (from bregma: posterior $4.0 \mathrm{~mm}$ and mediolateral $2.5 \mathrm{~mm}$ ), and two occipital (from bregma: posterior $8.0 \mathrm{~mm}$ and mediolateral $2.5 \mathrm{~mm}$ ). One subset of rats was implanted with a microdialysis probe in PFC (from bregma: anterior $3.0 \mathrm{~mm}$, mediolateral 0.5 $\mathrm{mm}$, ventral $4.0 \mathrm{~mm}$, and contralateral to the frontal EEG electrode) for microdialysis sample collection and local delivery of carbachol or NA, whereas the other subset was implanted with a microdialysis probe in parietal cortex (from bregma: posterior $3.6 \mathrm{~mm}$, mediolateral $2.6 \mathrm{~mm}$, ventral $2.0 \mathrm{~mm}$, and at an angle of 40 degrees) for microdialysis sample collection and local delivery of carbachol or NA. All stereotaxic coordinates were based on the rat atlas by Paxinos and Watson (2007). A limitation of the conventional EEG recording montage as used in the previous study (Pal et al., 2018) is that, in the absence of high-density spatial data, it allows only temporal complexity analysis or the measurement of the diversity of the EEG signal only in the temporal domain. To overcome this limitation and examine the changes in spatiotemporal complexity, in the current study, we surgically prepared another group of rats $(n=7)$ for intracranial high-density EEG recording (30 cortical sites). To the best of our knowledge, this approach to sample EEG data from across the cortical surface in combination with microdialysis delivery and sample collection has not been reported previously. The procedures for the surgical implantation of electrodes to record EEG and the guide tubes for microdialysis delivery of carbachol or NA as well as the chromatographic quantification of local acetylcholine levels have been described in detail in our previous study (Pal et al., 2018), and the same procedures were adopted for the high-density EEG cohort in the current study.

Experimental design. The experimental design for the EEG data collection before, during, and after sevoflurane anesthesia along with microdialysis delivery of carbachol or NA into prefrontal and parietal cortices is illustrated in Figure 1. The detailed experimental procedures have been described in our previous study (Pal et al., 2018). In brief, on the day of the experiment, the rats were connected to the EEG recording cable, and a microdialysis probe being continuously perfused $(2 \mu \mathrm{l} / \mathrm{min}$ using a CMA/400 syringe pump, CMA Microdialysis, Harvard Apparatus) with Ringer's solution (147 mM NaCl, $2.4 \mathrm{mM} \mathrm{CaCl}_{2}, 4.0 \mathrm{~mm} \mathrm{KCl,} 10 \mu \mathrm{M}$ 
neostigmine; $\mathrm{pH} 6.0 \pm 0.2$ ), was lowered into either prefrontal (CMA/11, 1-mm-long cuprophane membrane, 0.24-mm-diameter, $6 \mathrm{kDa}$ membrane cutoff) or parietal cortex (CMA/11, 2-mm-long cuprophane membrane, 0.24 -mm-diameter, $6 \mathrm{kDa}$ membrane cutoff) for carbachol or NA delivery and simultaneous collection of microdialysis samples. The EEG data were collected during baseline wake state for $75 \mathrm{~min}$. To hold the behavioral state constant during the baseline condition, the rats were kept awake by introduction of novel objects and gentle tapping on the recording chamber. At the completion of $75 \mathrm{~min}$ of baseline wake recording, sevoflurane exposure $(1.9 \%-2.4 \%)$ was started and titrated to maintain (1) loss of righting reflex, which is a widely accepted behavioral surrogate for anesthetic-induced unconsciousness in rats, and (2) highamplitude, slow-wave EEG. The anesthetic exposure was continued for $75 \mathrm{~min}$, after which either carbachol (5 mM) or NA ( $20 \mathrm{~mm})$ was reverse dialyzed for $12.5 \mathrm{~min}$ into the PFC ( $n=10$ rats for carbachol and $n=8$ rats for NA) or the parietal cortex $(n=11$ rats each for carbachol and NA) while the rats were still inhaling the same concentration of sevoflurane anesthesia. At the end of carbachol or NA delivery, the EEG recordings continued under sevoflurane anesthesia for another $50 \mathrm{~min}$. Thereafter, sevoflurane exposure was stopped and EEG data were collected for 37.5 min during the post-sevoflurane recovery wake state. The use of reverse dialysis delivery allowed us to restrict the spatial spread of carbachol or NA, thereby avoiding nonspecific effects associated with activation or inhibition of spatially distinct brain regions. Importantly, administration of carbachol and NA through reverse dialysis allowed us to simultaneously monitor the changes in local acetylcholine levels, which have been reported in our previous study (Pal et al., 2018). In the current study, the new cohort of male Sprague Dawley rats $(n=7)$ prepared for intracranial high-density EEG recordings was also implanted with a microdialysis probe in PFC for measuring acetylcholine levels during local reverse dialysis delivery of $5 \mathrm{~mm}$ carbachol. The following EEG segments, visually inspected and free of any artifacts, were selected for analysis: (1) baseline wake condition: last 300 s of the baseline wake state before exposure to sevoflurane anesthesia; (2) sevoflurane anesthesia: last $300 \mathrm{~s}$ of the sevoflurane exposure before pharmacological stimulation with carbachol or NA; (3) carbachol: $300 \mathrm{~s}$ from the point of first visible change in EEG after the start of carbachol delivery during sevoflurane anesthesia; (4) NA: 90-220 s from the point of first visible change in EEG after the start of NA delivery during sevoflurane anesthesia; and (5) post-sevoflurane recovery wake state: 300 s from the recovery of righting reflex, which occurred within $750 \mathrm{~s}$ after the cessation of sevoflurane anesthesia. The emergence time from sevoflurane anesthesia, as indicated by the return of righting reflex, was variable among rats, but all the rats recovered within $750 \mathrm{~s}$ of sevoflurane discontinuation. The epoch selection and the data analysis scheme for the high-density cohort were the same as that followed for the five-channel prefrontal-carbachol group described above and reported previously (Pal et al., 2018).

EEG recording. Monopolar EEG signals were recorded with reference to a stainless-steel electrode over nasal sinus, as has been reported previously in studies from our (Borjigin et al., 2013; Pal et al., 2015, 2016) and other (Castro et al., 2013, 2014) laboratories. The signals were amplified $(5000 \times)$ and bandpass filtered between 0.1 and $300 \mathrm{~Hz}$ using a Grass model 15 LT bipolar portable physiodata amplifier system (15A54 Quad Amplifier, Natus Neurology), and digitized at $1 \mathrm{kHz}$ using MP150 data acquisition unit (Acqknowledge software version 4.1.1, Biopac Systems). The high-density EEG dataset was recorded on a Scout Grapevine Neural Processor (Ripple Neuro) using a bandpass filter of $0.1-300 \mathrm{~Hz}$ and sampling rate of $1 \mathrm{kHz}$. The raw EEG data were exported into MATLAB (version 2015a; The MathWorks) and downsampled to $500 \mathrm{~Hz}$ (resample.m function in MATLAB signal processing toolbox) for further analysis.

Corticocortical coherence analysis. Corticocortical coherence was measured by the magnitude squared coherence method using the mscohere.m function in MATLAB signal processing toolbox (The MathWorks). The EEG data were segmented into nonoverlapped $10 \mathrm{~s}$ windows, which were further divided into $2 \mathrm{~s}$ subwindows with $80 \%$ overlap. Each subwindow was multiplied with a Hamming window, and the coherence was estimated from the cross-spectra and auto-spectra of the two EEG signals using Welch's averaged-modified periodogram method. The coherence for each window was estimated as a function of frequency $(0.5-250 \mathrm{~Hz})$ and between each pair of the five EEG channels. The mean global coherence was obtained by averaging the coherence for individual channel pairs for each animal in the following gamma frequency bands: low gamma $(25-55 \mathrm{~Hz})$, medium gamma $(85-125 \mathrm{~Hz})$, and high gamma $(125-155 \mathrm{~Hz})$. The choice of these frequency bands was based on our recent rat study in which we demonstrated high gamma (85-155 Hz) corticocortical coherence and frontal-parietal connectivity as a correlate of wakefulness that is disrupted during general anesthesia and sleep (Pal et al., 2016).

Frontal-parietal directed connectivity: normalized symbolic transfer entropy analysis. Normalized symbolic transfer entropy is an information theoretic measure that is considered a surrogate for directed cortical communication. Our previous findings with normalized symbolic transfer entropy as a surrogate for frontal-parietal directed connectivity changes in human subjects (Lee et al., 2009, 2013; Ku et al., 2011) and rats (Borjigin et al., 2013; Pal et al., 2016; Li et al., 2017) have been supported by reports from other laboratories that have used methodologically different approaches (Boly et al., 2012; Jordan et al., 2013). In the current study, we analyzed the EEG data for changes in directed connectivity between ipsilateral frontal and parietal channels in the gamma frequency bandwidth: low gamma $(25-55 \mathrm{~Hz})$, medium gamma $(85-125 \mathrm{~Hz})$, and high gamma $(125-155 \mathrm{~Hz})$. We used a Butterworth filter of order 4 (butter.m and filtfilt.m, MATLAB signal processing toolbox) to filter the raw EEG data for the frequency bands of interest (i.e., low, medium, and high gamma), and segmented the filtered data into nonoverlapped $10 \mathrm{~s}$ windows. The calculation of normalized symbolic transfer entropy requires three parameters: embedding dimension, time delay, and prediction time. We fixed the embedding dimension at 3 , and time delay at 5, 2, and 1 , corresponding to low, medium, and high gamma, respectively. For each window, we searched the prediction time between 1 and 50 (corresponding to $2-100 \mathrm{~ms}$ ) and selected the one that yielded maximum normalized symbolic transfer entropy in the frontal-to-parietal and parietal-to-frontal direction. These parameters for normalized symbolic transfer entropy have been used in our previous rat studies (Borjigin et al., 2013; Pal et al., 2016; Li et al., 2017).

Spectral power analysis. The absolute power for the slow EEG oscillations was assessed in the temporal domain by applying a bandpass filter $(0.5-1 \mathrm{~Hz})$ to the EEG data using the eegfiltnew function in the EEGLAB toolbox (Delorme and Makeig, 2004). The power values were calculated for each nonoverlapped $10 \mathrm{~s}$ window, and the averaged power across all the windows and all the available channels was computed.

Temporal Lempel-Ziv complexity analysis. Lempel-Ziv complexity computes the complexity of data with finite length sequences (Lempel and Ziv, 1976; Ziv and Lempel, 1977, 1978) and has been shown to be a valuable tool to investigate the neurophysiological changes associated with states of consciousness (Casali et al., 2013; Abásolo et al., 2015; Schartner et al., 2015, 2017a,b; Hudetz et al., 2016; Li and Mashour, 2019). Because of the limited number of EEG channels (Pal et al., 2018), we could not compute the spatial complexity and therefore focused on the temporal complexity in ipsilateral frontal and parietal channels. The EEG signals were detrended using local linear regression with a $10 \mathrm{~s}$ window at a 5 s overlap (locdetrend function in Chronux analysis software), and lowpass filtered at $175 \mathrm{~Hz}$ via Butterworth filter of order 5 (butter and filtfilt functions in MATLAB signal processing toolbox). The instantaneous amplitude was calculated from the Hilbert transform of the signal, which was then binarized using its mean value as the threshold for each channel (Schartner et al., 2017a). The binary sequence was segmented into nonoverlapped $10 \mathrm{~s}$ windows. For each of these $10 \mathrm{~s}$ windows, the Lempel-Ziv complexity algorithm searches for the instances of consecutive characters or "words" and counts the number of times a new word is encountered. To assess the degree to which the difference in the complexity across the states is not due to spectral changes, we generated surrogate data through phase randomization while preserving the spectral profiles of the signal (Schartner et al., 2015, 2017a), and normalized the original Lempel-Ziv complexity by the mean of the Lempel-Ziv complexity values from $N=50$ surrogate time series. The resultant normalized Lempel-Ziv complexity values reflect complexity beyond the spectral changes, which were then averaged across all the windows as the estimate of the complexity at each studied state. 
Spatiotemporal Lempel-Ziv complexity anal$y$ sis. To overcome the limitations of low channel count (five) and to reveal the complexity changes in terms of spatial diversity, we implanted a new cohort of rats $(n=7)$ in the current study for high-density EEG recordings. The spatial distribution of electrodes (30) covering the entire cortex allowed us to quantify spatiotemporal complexity, and in addition served as an important control group to ascertain the validity of the temporal complexity analysis conducted on five-channel EEG data. The EEG data in 3 of 7 rats in the high-density cohort were not useable because of excessive artifacts and noise levels. After excluding these rats and any bad channels, the remaining EEG signals (number of channels: 25-29) were detrended, lowpass filtered at $175 \mathrm{~Hz}$, and divided into nonoverlapped $2 \mathrm{~s}$ windows after removing the common signal across channels. For each window, the instantaneous amplitude was estimated by applying the Hilbert transform (Schartner et al., 2015, 2017a), which was binarized using its mean value as the threshold for each channel. The data were then converted into a binary matrix, in which rows represent channels and columns represent time points. The complexity of the spatiotemporal matrix was assessed by Lempel-Ziv complexity (Casali et al., 2013; Li and Mashour, 2019), which searches the binary matrix, time point by time point, and counts the number of different spatial patterns across different time points. We then normalized the spatiotemporal complexity by the mean of those from the surrogate data through phase randomization, to examine whether the difference in the spatiotemporal complexity across the states is due to spectral changes.

Statistical analyses. Statistical analyses were conducted in consultation with the Consulting for Statistics, Computing and Analytics Research unit at the University of Michigan (Ann Arbor, Michigan). The initial study (Pal et al., 2018) from which the EEG data were used in the current study was designed to have $80 \%$ power at $\alpha$ of 0.05 . All statistical comparisons were conducted in a within-group design using the programming and statistical language $\mathrm{R}$ (version 3.6.0) (R Core Team, 2019). We used a linear mixed model with random intercept for each rat. This accounts for the correlations among observations of the same rat and allows unified reporting of all pairwise state comparisons. Post hoc pairwise tests were single-step corrected for multiple comparisons (Package multicomp). Models were fit with restricted maximum likelihood. $p$ values of $<0.05$ were considered statistically significant. For clarity and readability, we have provided the $p$ values in Results, whereas the mean, SD, F statistics, and effect sizes for all datasets and comparisons (except the acetylcholine dataset) have been provided in tabular format, as referenced in Results. The acetylcholine dataset has relatively fewer comparisons because of which we have reported the associated statistical values in the text in Results.

\section{Results}

Corticocortical gamma coherence does not correlate with level of consciousness

Carbachol delivery into prefrontal and parietal cortices

Compared with the baseline wake state, sevoflurane anesthesia was characterized by a significant reduction $(p \leq 0.001)$ in the

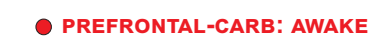

$\triangle$ PARIETAL-CARB: NOT AWAKE A

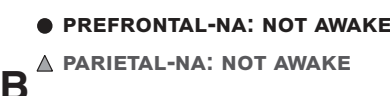

B

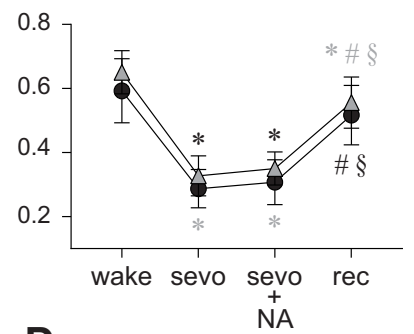

D
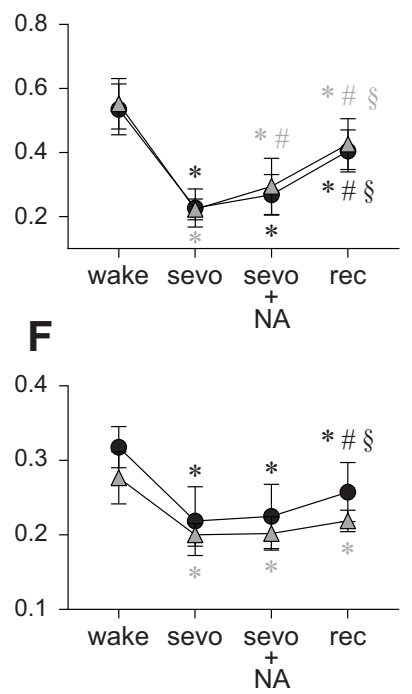

Figure 2. Corticocortical coherence does not correlate with level of consciousness. $\boldsymbol{A}-\boldsymbol{F}$, Sevoflurane anesthesia (sevo) signifi(Pal et al., 2018) but failed to restore gamma coherence (high, medium, low) to baseline wake dhe post hoc pairwise tests were single-step corrected for multiple comparisons. The statistical comparisons are shown at $p<$ ificance symbols are color-coded to match the line-symbol plots. *Significant compared with baseline wake state. "Significant compared with sevo. §Significant compared with CARB or NA during sevo.

corticocortical coherence across the gamma bandwidth in both the prefrontal and parietal cortical groups (Fig. 2 A, C,E; Table 1). Carbachol delivery into PFC of sevoflurane-anesthetized rats was shown to restore wakefulness despite the continuous presence of sevoflurane anesthesia (Pal et al., 2018). However, analysis of the EEG from the same epoch showed that, despite the restoration of wakefulness, the corticocortical gamma coherence (high, medium, low) was not statistically different from that observed during sevoflurane anesthesia ( $p \geq 0.74$ ), and remained significantly reduced ( $p \leq 0.0017$ ) compared with the baseline wake state (Fig. $2 A, C, E$; Table 1). Wakefulness was determined based on clear attempts at righting or recovery of the righting reflex. All rats in the prefrontal carbachol group showed such behavioral responses and 4 of 11 rats regained complete mobility. Detailed descriptions of behavioral and physiological changes, as well as a representative movie clip showing completely mobile animal after carbachol delivery, are provided in our previous publication (Pal et al., 2018). As opposed to the effect of carbachol in PFC, the 
Table 1. Corticocortical coherence before, during, and after sevoflurane administration and carbachol delivery into prefrontal and parietal cortices

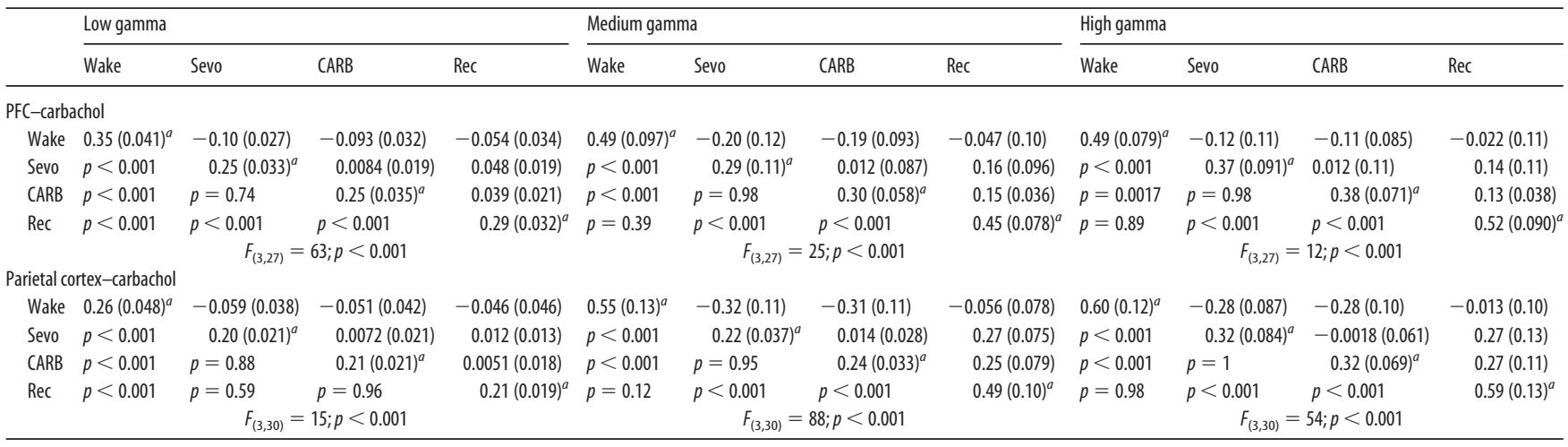

${ }^{a}$ Mean ( \pm SD) for each state is shown along the diagonal of each section.

The cells above the mean ( \pm SD) values show the effect sizes as the difference of the means ( \pm SD). Sevo, Sevoflurane administration; CARB, carbachol delivered during sevo; Rec, post-sevoflurane recovery wake.

Table 2. Corticocortical coherence before, during, and after sevoflurane administration and NA delivery into prefrontal and parietal cortices

\begin{tabular}{|c|c|c|c|c|c|c|c|c|c|c|c|c|}
\hline & \multicolumn{4}{|l|}{ Low gamma } & \multicolumn{4}{|c|}{ Medium gamma } & \multicolumn{4}{|l|}{ High gamma } \\
\hline & Wake & Sevo & NA & Rec & Wake & Sevo & NA & Rec & Wake & Sevo & NA & $\operatorname{Rec}$ \\
\hline \multicolumn{13}{|l|}{ PFC-NA } \\
\hline Sevo & $p<0.001$ & $0.22(0.046)^{a}$ & $0.0063(0.014)$ & $0.039(0.029)$ & $p<0.001$ & $0.23(0.059)^{a}$ & $0.041(0.036)$ & $0.18(0.061)$ & $p<0.001$ & $0.29(0.060)^{a}$ & $0.020(0.035)$ & $0.23(0.096)$ \\
\hline NA & $p<0.001$ & $p=0.87$ & $0.23(0.043)^{a}$ & $0.033(0.025)$ & $p<0.001$ & $p<0.26$ & $0.27(0.063)^{a}$ & $0.14(0.068)$ & $p<0.001$ & $p=0.94$ & $0.31(0.070)^{a}$ & $0.21(0.089)$ \\
\hline $\operatorname{Rec}$ & $p<0.001$ & $p<0.001$ & $p<0.001$ & $0.26(0.040)^{a}$ & $p<0.001$ & $p<0.001$ & $p<0.001$ & $0.41(0.066)^{a}$ & $p=0.14$ & $p<0.001$ & $p<0.001$ & $0.52(0.093)^{a}$ \\
\hline & \multicolumn{4}{|c|}{$F_{(3,21)}=61 ; p<0.001$} & \multicolumn{4}{|c|}{$F_{(3,21)}=78 ; p<0.001$} & \multicolumn{4}{|c|}{$F_{(3,21)}=36 ; p<0.001$} \\
\hline Sevo & $p<0.001$ & $0.20(0.015)^{a}$ & $0.0019(0.024)$ & $0.019(0.018)$ & $p<0.001$ & $0.22(0.033)^{a}$ & 0.0720 .083 & $0.21(0.083)$ & $p<0.001$ & $0.33(0.062)^{a}$ & $0.023(0.064)$ & $0.23(0.11)$ \\
\hline NA & $p<0.001$ & $p=1$ & $0.20(0.22)^{a}$ & $0.017(0.021)$ & $p<0.001$ & $p=0.021$ & $0.29(0.088)^{a}$ & $0.13(0.065)$ & $p<0.001$ & $p=0.84$ & $0.35(0.052)^{a}$ & $0.21(0.079)$ \\
\hline Rec & $p<0.001$ & $p=0.11$ & $p=0.17$ & $0.22(0.014)^{a}$ & $p<0.001$ & $p<0.001$ & $p<0.001$ & $0.43(0.079)^{a}$ & $p=0.0036$ & $p<0.001$ & $p<0.001$ & $0.56(0.080)^{a}$ \\
\hline & \multicolumn{4}{|c|}{$F_{(3,30)}=38 ; p<0.001$} & \multicolumn{4}{|c|}{$F_{(3,30)}=69 ; p<0.001$} & \multicolumn{4}{|c|}{$F_{(3,30)}=65 ; p<0.001$} \\
\hline
\end{tabular}

${ }^{a}$ Mean ( \pm SD) for each state is shown along the diagonal of each section.

The cells above the mean ( \pm SD) values show the effect sizes as the difference of the means ( \pm SD). Sevo, Sevoflurane administration; NA, NA delivered during sevo; Rec, post-sevoflurane recovery wake.

reverse dialysis delivery of carbachol into parietal cortex of sevoflurane-anesthetized rat was ineffective in restoring wakefulness (Pal et al., 2018) and analysis of the EEG during the same epoch showed that coherence across the gamma bandwidth remained significantly low $(p<0.001)$ compared with the baseline wake state (Fig. $2 A, C$,E; Table 1); there was no significant difference in coherence in any of the gamma bands between the sevoflurane anesthesia epoch and the carbachol delivery epoch $(p \geq$ 0.88 ). The post-sevoflurane recovery wake state in the prefrontal and parietal groups was characterized by increase in coherence in high gamma ( $p \leq 0.001$ compared with sevoflurane, $p<0.001$ compared with carbachol epochs) and medium gamma $(p<$ 0.001 compared with sevoflurane, $p<0.001$ compared with carbachol epochs) bands, which returned to the baseline wake levels ( $p \geq 0.12$ ) (Fig. $2 A, C$; Table 1 ). The low gamma coherence during post-sevoflurane recovery wake state in both prefrontal and parietal groups remained significantly below $(p<0.001)$ than that observed during the baseline wake state (Fig. 2E; Table 1), even though there was a significant increase in the PFC group compared with both sevoflurane $(p<0.001)$ and carbachol delivery $(p<0.001)$ epochs (Fig. 2E; Table 1$)$.

NA delivery into prefrontal and parietal cortices

Sevoflurane anesthesia produced a significant reduction $(p<$ 0.001 ) in the corticocortical coherence across the gamma bandwidth (Fig. 2 B, D,F; Table 2). Reverse dialysis delivery of NA into either prefrontal or parietal cortex during sevoflurane anesthesia was not observed to produce any signs of wakefulness (Pal et al.,
2018). During the same epoch (NA delivery), the corticocortical coherence in high gamma $(p<0.001)$ and medium gamma $(p<$ 0.001 ) bands in both prefrontal and parietal groups remained significantly reduced compared with the baseline wake state (Fig. $2 B, D$; Table 2 ), even though there was a significant increase ( $p=$ 0.021 compared with sevoflurane) in medium gamma coherence in the parietal cortex group. The post-sevoflurane recovery wake state in both prefrontal and parietal groups was also characterized by a significant increase in high and medium gamma coherence $(p<0.001)$ compared with both sevoflurane and NA epochs (Fig. 2B,D; Table 2). Although the increase in high gamma coherence during post-sevoflurane recovery period in the prefrontal group reached the baseline levels and was not statistically different from the wake state $(p=0.14)$, the high gamma coherence in parietal group remained significantly lower than the baseline levels ( $p=$ $0.0036)$. Similarly, the medium gamma coherence during the postsevoflurane recovery period, in both prefrontal and parietal groups, remained significantly lower $(p<0.001)$ than the baseline wake levels. The low gamma coherence during post-sevoflurane recovery wake state in the prefrontal group increased compared with sevoflurane $(p<0.001)$ and NA delivery $(p<0.001)$ epochs but still remained significantly low $(p<0.001)$ compared with baseline wake state (Fig. 2F; Table 2). In the parietal group, the low gamma coherence during the post-sevoflurane epoch remained significantly low $(p<0.001)$ compared with the baseline wake levels and was not significantly different from either sevoflurane $(p=0.11)$ or NA $(p=$ 0.17) epochs (Fig. 2F; Table 2). 
Directed cortical gamma connectivity does not correlate with level of consciousness

Carbachol delivery into prefrontal and parietal cortices

Compared with the baseline wake state, sevoflurane anesthesia was characterized by a significant suppression of bidirectional (frontal-to-parietal and parietal-tofrontal) connectivity in the high and medium gamma bands $(p \leq 0.020)$ in both prefrontal and parietal groups (Figs. $3 A, C, 4 A, C$; Tables 3, 4). The delivery of carbachol into prefrontal, but not parietal cortex, was earlier shown to restore wakefulness in the presence of sevoflurane anesthesia (Pal et al., 2018). However, bidirectional frontal-parietal gamma connectivity was not restored after delivery of carbachol into either PFC (restored wakefulness) or parietal cortex (no signs of wakefulness). High and medium gamma connectivity remained significantly below the baseline wake levels (high gamma: $p<$ 0.0074; medium gamma: $p<0.001$ ) and was not significantly different from that observed during sevoflurane anesthesia ( $p \geq 0.070$ ) (Figs. $3 A, C, 4 A, C$; Tables 3 , $4)$. In both the prefrontal and parietal groups, the post-sevoflurane recovery wake state was characterized by a significant increase in high gamma and medium gamma bidirectional frontal-parietal connectivity $(p<0.001$ compared with both sevoflurane and carbachol epochs) (Figs. $3 A, C, 4 A, C$; Tables 3,4 ); the connectivity levels in both the groups returned to baseline wake levels $(p \geq 0.1)$. The bidirectional frontal-parietal connectivity in low gamma bandwidth showed widely variable effects (Figs. 3E, 4E; Tables 3, 4).

\section{NA delivery into prefrontal and}

parietal cortices

Sevoflurane anesthesia was characterized

by a significant suppression of bidirectional (frontal-to-parietal and parietal-to-frontal) connectivity in the high and medium gamma bands $(p<0.001)$ in both prefrontal and parietal groups (Figs. 3 B, D, $4 B, D$; Tables 5, 6). NA delivery into the prefrontal or parietal cortex of sevoflurane-anesthetized rats did not produce any signs of wakefulness (Pal et al., 2018), and the analysis of the concomitant EEG showed no significant change in the high gamma or medium gamma bidirectional frontal-parietal connectivity ( $p \geq 0.85$ compared with sevoflurane anesthesia), which remained significantly below the baseline wake levels $(p<0.001)$ (Figs. $3 B, D, 4 B, D$; Tables 5,6 ). The post-sevoflurane recovery wake epoch in both prefrontal and parietal groups was characterized by a significant increase in bidirectional frontal-parietal connectivity in both high gamma $(p<0.001$ compared with sevoflurane anesthesia and NA epochs) and medium gamma ( $p \leq 0.029$ compared with sevoflurane anesthesia, $p \leq 0.0094$ compared with NA) bands (Figs. $3 B, D, 4 B, D$; Tables 5, 6); the high and medium gamma bidirectional connectivity in the
- PREFRONTAL-CARB: AWAKE $\triangle$ PARIETAL-CARB: NOT AWAKE A PRTEL-CARB: NOT AWAKE
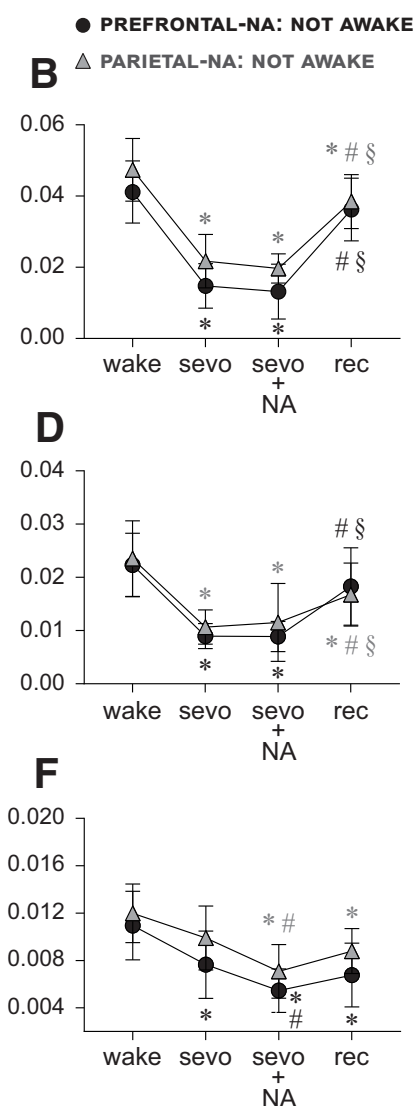

Figure 3. Frontal-to-parietal directed gamma connectivity does not correlate with level of consciousness. A-D, Frontal-toparietal connectivity in the high $(125-155 \mathrm{~Hz})$ and medium $(85-125 \mathrm{~Hz})$ gamma bands was significantly reduced during sevoflu-

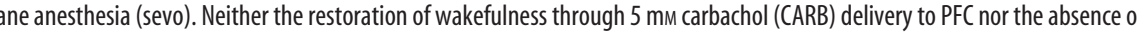
al et al., 2018), had any significant effect on the high and medium gamma frontal-to-parietal connectivity, which remained

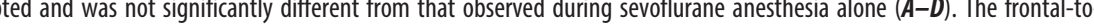
medium gamma bands for all conditions, except in the parietal cortex-NA group $(\boldsymbol{B}, \boldsymbol{D})$, where it remained significantly lower mixed model with random intercept for each rat was used for the statistical comparisons, and the post hoc pairwise tests were single-step corrected for multiple comparisons. The statistical comparisons are shown at $p<0.05$. The mean, SD, exact $p$ values, color-coded to match the line-symbol plots. *Significant compared with wake. "Significant compared with sevo. §Significant compared with CARB or NA during sevo.

prefrontal group returned to baseline wake levels ( $p \geq 0.077)$ but in the parietal cortex group it remained significantly below $(p \leq 0.019$ ) the baseline wake levels (Figs. $3 B, D, 4 B, D$; Tables $5,6)$. The bidirectional frontal-parietal connectivity in low gamma bandwidth showed widely variable effects (Figs. $3 F$, $4 F$; Tables 5, 6).

\section{Spectral power in slow oscillations does not correlate with level of consciousness \\ Carbachol delivery into prefrontal and parietal cortices}

As expected, compared with the baseline wake state, sevoflurane anesthesia was associated with a significant increase $(p \leq 0.02)$ in spectral power in slow oscillations (Fig. $5 A$; Table 7 ). In our previous report (Pal et al., 2018), we showed that the delivery of carbachol into PFC of sevoflurane-anesthetized rats could restore wakefulness and produce EEG activation, while carbachol into parietal cortex produced only EEG activation (Pal et al., 2018). Analysis of the carbachol epoch showed that regardless of the presence (stimulation of PFC) or absence (stimulation of parietal 
cortex) of wakefulness, the EEG activation was accompanied by a significant decrease in slow oscillations power compared with the sevoflurane epoch ( $p \leq 0.001$ for carbachol in the PFC, $p<0.001$ for carbachol in the parietal cortex) (Fig. 5A; Table 7). The post-sevoflurane recovery wake period in both the prefrontal and parietal groups was also characterized by decrease in the spectral power in slow oscillations that was significantly lower than that observed during sevoflurane anesthesia $(p=$ 0.0018 for carbachol in the PFC, $p<0.001$ for carbachol in the parietal cortex). For both the prefrontal and parietal cortical groups, there was no significant difference $(p \geq 0.23)$ in the spectral power between baseline wake state, carbachol-induced EEG activation, and the post-sevoflurane recovery wake period (Fig. $5 A$; Table 7 ).

\section{NA delivery into prefrontal and}

parietal cortices

Sevoflurane anesthesia produced a significant increase $(p \leq 0.0051)$ in the spectral power in slow oscillations in both the prefrontal and parietal cortical groups (Fig. $5 B$; Table 7 ). NA in prefrontal or parietal cortex did not restore wakefulness during ongoing sevoflurane administration, but it was shown to produce EEG activation (Pal et al., 2018). These findings are consistent with a previous study that demonstrated EEG activation without behavioral arousal in propofol-anesthetized rats after systemic treatment with a noradrenergic reuptake blocker (Kenny et al., 2015). The NA epoch, characterized by EEG activation, was also marked by a significant decrease in the spectral power in slow oscillations in both the prefrontal and parietal groups $(p<0.001$ compared with sevoflurane anesthesia) (Fig. 5B; Table 7). The power in slow oscillations in the postsevoflurane recovery wake period in both prefrontal and parietal groups was significantly reduced $(p<$ 0.001) compared with sevoflurane anesthesia epoch (Fig. 5B; Table 7). For both the prefrontal and parietal cortical groups, there was no significant difference $(p \geq 0.56)$ in the spectral power between the post-sevoflurane recovery wake period, the NAinduced EEG activation, and the baseline wake state (Fig. $5 B$; Table 7).

\section{Electroencephalographic temporal Lempel-Ziv complexity does not correlate with level of consciousness Carbachol delivery into prefrontal and parietal cortices} The temporal complexity was measured over the frontal and parietal areas. As compared with the baseline wake state, sevoflurane anesthesia was characterized by a significant reduction $(p<$ 0.001 ) in the temporal complexity (Fig. 6A, C; Table 8). Carbachol delivery into PFC (restoration of wakefulness) or parietal cortex (no behavioral arousal) during sevoflurane anesthesia produced EEG activation, which was characterized by increase in temporal complexity ( $p<0.001$ compared with sevoflurane anesthesia) (Fig. 6A, $C$; Table 8). In both the prefrontal and parietal groups, the post-sevoflurane recovery wake period was characterized by high temporal complexity in frontal (Fig. 6A; Table 8) and parietal areas (Fig. $6 C$; Table 8$)(p<0.001$ compared with sevoflurane anesthesia), which was not significantly different ( $p \geq 0.28$ ) from that observed during the baseline wake state and the carbachol delivery epoch (Fig. 6A,C; Table 8).

NA delivery into prefrontal and parietal cortices

Compared with the baseline wake state, the temporal complexity was significantly reduced during sevoflurane anesthesia $(p<$ 0.001) (Fig. 6B,D; Table 9). NA delivery into PFC or parietal cortex of sevoflurane anesthetized rats did not produce any signs of wakefulness, but it did produce EEG activation (Pal et al., 2018). Analysis of the NA epoch in both the prefrontal and parietal cortical groups showed a significant increase in the temporal complexity over the frontal $(p<0.001)$ (Fig. 6B; Table 9) and parietal $(p<0.001)$ (Fig. $6 D$; Table 9 ) areas, compared with 
Table 3. Frontal-to-parietal connectivity before, during, and after sevoflurane administration and carbachol delivery into prefrontal and parietal cortices

\begin{tabular}{|c|c|c|c|c|c|c|c|c|c|c|c|c|}
\hline & \multicolumn{4}{|l|}{ Low gamma } & \multicolumn{4}{|l|}{ Medium gamma } & \multicolumn{4}{|l|}{ High gamma } \\
\hline & Wake & Sevo & CARB & $\operatorname{Rec}$ & Wake & Sevo & CARB & $\operatorname{Rec}$ & Wake & Sevo & CARB & $\operatorname{Rec}$ \\
\hline \multicolumn{13}{|c|}{ PFC-carbachol } \\
\hline Wake & $0.013(0.0042)^{a}$ & $-0.0026(0.0030)$ & $-0.0039(0.0040)$ & $-0.0039(0.0031)$ & $0.022(0.0045)^{a}$ & $-0.0075(0.0058)$ & $-0.0084(0.0054)$ & $-44 \mathrm{e}-5(0.0044)$ & $0.037(0.0079)^{a}$ & $-0.0089(0.0082)$ & $-0.010(0.010)$ & $0.0056(0.010)$ \\
\hline Sevo & $p=0.044$ & $0.011(0.0032)^{a}$ & $-0.0014(0.0020)$ & $-0.0013(0.0026)$ & $p<0.001$ & $0.014(0.0034)^{a}$ & $-93 e-5(0.0041)$ & $0.0071(0.0050)$ & $p=0.020$ & $0.028(0.0069)^{a}$ & $-93 e-5(0.011)$ & $0.015(0.012)$ \\
\hline CARB & $p<0.001$ & $p=0.51$ & $0.0095(0.0034)^{a}$ & $38 \mathrm{e}-6(0.0035)$ & $p<0.001$ & $p=0.92$ & $0.014(0.0025)^{a}$ & $0.0080(0.0023)$ & $p=0.0074$ & $p=1$ & $0.027(0.0060)^{a}$ & $0.015(0.0067)$ \\
\hline $\operatorname{Rec}$ & $p<0.001$ & $p=0.54$ & $p=1$ & $0.010(0.0034)^{a}$ & $p=1$ & $p<0.001$ & $p<0.001$ & $0.022(0.0032)^{a}$ & $p=0.27$ & $p<0.001$ & $p<0.001$ & $0.043(0.0082)^{c}$ \\
\hline & \multicolumn{4}{|c|}{$F_{(3,27)}=7.0 ; p=0.0012$} & \multicolumn{4}{|c|}{$F_{(3,27)}=19 ; p<0.001$} & \multicolumn{4}{|c|}{$F_{(3,27)}=12 ; p<0.001$} \\
\hline \multicolumn{13}{|c|}{ Parietal cortex-carbachol } \\
\hline Wake & $0.014(0.0047)^{a}$ & $-0.0015(0.0028)$ & $-0.0017(0.0041)$ & $-0.0022(0.0032)$ & $0.029(0.0067)^{a}$ & $-0.016(0.0069)$ & $-0.020(0.0081)$ & $-0.0034(0.0075)$ & $0.050(0.0082)^{a}$ & $-0.028(0.010)$ & $-0.033(0.0095)$ & $-0.0022(0.011)$ \\
\hline Sevo & $p=0.41$ & $0.013(0.0043)^{a}$ & $-24 \mathrm{e}-5(0.0034)$ & $-69 \mathrm{e}-5(0.0023)$ & $p<0.001$ & $0.013(0.0022)^{a}$ & $-0.0047(0.0027)$ & $0.012(0.0060)$ & $p<0.001$ & $0.022(0.0086)^{a}$ & -0.00490 .0065 & $0.026(0.014)$ \\
\hline CARB & $p=0.28$ & $p=1$ & $0.013(0.0063)^{a}$ & $-45 e-5(0.0032)$ & $p<0.001$ & $p=0.070$ & $0.0082(0.0023)^{a}$ & $0.017(0.0056)$ & $p<0.001$ & $p=0.43$ & $0.017(0.0044)^{a}$ & $0.031(0.011)$ \\
\hline $\operatorname{Rec}$ & $p=0.11$ & $p=0.89$ & $p=0.97$ & $0.012(0.0060)^{a}$ & $p=0.28$ & $p<0.001$ & $p<0.001$ & $0.025(0.0056)^{a}$ & $p=0.91$ & $p<0.001$ & $p<0.001$ & $0.048(0.010)^{a}$ \\
\hline & \multicolumn{4}{|c|}{$F_{(3,30)}=1.9 ; p=0.15$} & \multicolumn{4}{|c|}{$F_{(3,30)}=51 ; p<0.001$} & \multicolumn{4}{|c|}{$F_{(3,30)}=56 ; p<0.001$} \\
\hline
\end{tabular}

${ }^{a}$ Mean ( \pm SD) for each state is shown along the diagonal of each section.

The cells above the mean ( \pm SD) values show the effect sizes as the difference of the means ( \pm SD). Sevo, Sevoflurane administration; CARB, carbachol delivered during sevo; Rec, post-sevoflurane recovery wake.

Table 4. Parietal-to-frontal connectivity before, during, and after sevoflurane administration and carbachol delivery into prefrontal and parietal cortices

\begin{tabular}{|c|c|c|c|c|c|c|c|c|c|c|c|c|}
\hline & \multicolumn{4}{|l|}{ Low gamma } & \multicolumn{4}{|l|}{ Medium gamma } & \multicolumn{4}{|l|}{ High gamma } \\
\hline & Wake & Sevo & CARB & $\operatorname{Rec}$ & Wake & Sevo & CARB & $\operatorname{Rec}$ & Wake & Sevo & CARB & $\operatorname{Rec}$ \\
\hline \multicolumn{13}{|c|}{ PFC-carbachol } \\
\hline Wake & $0.011(0.0034)^{a}$ & $-0.0015(0.023)$ & $-0.0023(0.0029)$ & $-0.0029(0.0019)$ & $0.025(0.0053)^{a}$ & $-0.0081(0.0068)$ & $-0.0088(0.0058)$ & $-11 e-5(0.0054)$ & $0.038(0.0086)^{a}$ & $-0.0093(0.0087)$ & $-0.0099(0.0090)$ & $0.0065(0.0091)$ \\
\hline Sevo & $p=0.18$ & $0.0099(0.0025)^{a}$ & $-83 e-5(0.0018)$ & $-0.0014(0.0022)$ & $p<0.001$ & $0.017(0.0053)^{a}$ & $-66 e-5(0.0054)$ & $0.0080(0.0064)$ & $p=0.0077$ & $0.028(0.0068)^{a}$ & $-58 \mathrm{e}-5(0.011)$ & $0.016(0.011)$ \\
\hline CARB & $p=0.0077$ & $p=0.66$ & $0.0091(0.0022)^{a}$ & $-58 \mathrm{e}-5(0.0025)$ & $p<0.001$ & $p=0.98$ & $0.016(0.0031)^{a}$ & $0.0087(0.0020)$ & $p=0.0038$ & $p=1$ & $0.028(0.0065)^{a}$ & $0.017(0.0046)$ \\
\hline $\operatorname{Rec}$ & $p<0.001$ & $p=0.21$ & $p=0.85$ & $0.0085(0.0029)^{a}$ & $p=1$ & $p<0.001$ & $p<0.001$ & $0.025(0.0039)^{a}$ & $p=0.11$ & $p<0.001$ & $p<0.001$ & $0.044(0.0070)^{a}$ \\
\hline & \multicolumn{4}{|c|}{$F_{(3,27)}=6.0 ; p=0.0029$} & \multicolumn{4}{|c|}{$F_{(3,27)}=16 ; p<0.001$} & \multicolumn{4}{|c|}{$F_{(3,27)}=15 ; p<0.001$} \\
\hline \multicolumn{13}{|c|}{ Parietal cortex-carbachol } \\
\hline Wake & $0.013(0.0045)^{a}$ & $55 e-5(0.0016)$ & $-0.0021(0.0025)$ & $-0.0011(0.0030)$ & $0.031(0.0052)^{a}$ & $-0.016(0.0041)$ & $-0.019(0.0064)$ & $-0.0030(0.0060)$ & $0.051(0.0084)^{a}$ & $-0.028(0.011)$ & $-0.031(0.011)$ & $-0.0011(0.012)$ \\
\hline Sevo & $p=0.88$ & $0.013(0.0048)^{a}$ & $-0.0026(0.0024)$ & $-0.0017(0.0025)$ & $p<0.001$ & $0.015(0.0020)^{a}$ & $-0.0030(0.0035)$ & $0.013(0.0053)$ & $p<0.001$ & $0.023(0.0086)^{a}$ & $-0.0039(0.0071)$ & $0.026(0.016)$ \\
\hline CARB & $p=0.031$ & $p=0.0028$ & $0.011(0.0047)^{a}$ & $92 e-5(0.0028)$ & $p<0.001$ & $p=0.26$ & $0.012(0.0028)^{a}$ & $0.016(0.0060)$ & $p<0.001$ & $p=0.71$ & $0.019(0.0052)^{a}$ & $0.030(0.013)$ \\
\hline $\operatorname{Rec}$ & $p=0.43$ & $p=0.11$ & $p=0.61$ & $0.012(0.0060)^{a}$ & $p=0.23$ & $p<0.001$ & $p<0.001$ & $0.028(0.0058)^{a}$ & $p=1$ & $p<0.001$ & $p<0.001$ & $0.050(0.011)^{a}$ \\
\hline & \multicolumn{4}{|c|}{$F_{(3,30)}=4.9 ; p=0.0072$} & \multicolumn{4}{|c|}{$F_{(3,30)}=71 ; p<0.001$} & \multicolumn{4}{|c|}{$F_{(3,30)}=43 ; p<0.001$} \\
\hline
\end{tabular}

${ }^{a}$ Mean ( \pm SD) for each state is shown along the diagonal of each section.

The cells above the mean $( \pm S D$ ) values show the effect sizes as the difference of the means ( \pm SD). Sevo, Sevoflurane administration; CARB, carbachol delivered during sevo; Rec, post-sevoflurane recovery wake.

Table 5. Frontal-to-parietal connectivity before, during, and after sevoflurane administration and NA delivery into prefrontal and parietal cortices

\begin{tabular}{|c|c|c|c|c|c|c|c|c|c|c|c|c|}
\hline & \multicolumn{4}{|l|}{ Low gamma } & \multicolumn{4}{|l|}{ Medium gamma } & \multicolumn{4}{|l|}{ High gamma } \\
\hline & Wake & Sevo & NA & $\operatorname{Rec}$ & Wake & Sevo & NA & $\operatorname{Rec}$ & Wake & Sevo & NA & $\operatorname{Rec}$ \\
\hline \multicolumn{13}{|l|}{ PFC-NA } \\
\hline Wake & $0.011(0.0029)^{a}$ & $-0.0033(0.0014)$ & $-0.0055(0.0019)$ & $-0.0042(0.0014)$ & $0.022(0.0060)^{a}$ & $-0.013(0.0062)$ & $-0.013(0.0081)$ & $-0.0040(0.0087)$ & $0.041(0.0087)^{a}$ & $-0.026(0.014)$ & $-0.028(0.014)$ & $-0.0049(0.011)$ \\
\hline Sevo & $p<0.001$ & $0.0076(0.0028)^{a}$ & $-0.0022(0.0013)$ & $-87 \mathrm{e}-5(0.0016)$ & $p<0.001$ & $0.0090(0.0024)^{a}$ & $-83 e-6(0.0024)$ & $0.0093(0.0075)$ & $p<0.001$ & $0.015(0.0063)^{a}$ & $-0.0016(0.0038)$ & $0.021(0.0084)$ \\
\hline NA & $p<0.001$ & $p<0.001$ & $0.0055(0.0018)^{a}$ & $0.0013(0.0014)$ & $p<0.001$ & $p=1$ & $0.0089(0.0028)^{a}$ & $0.0094(0.0082)$ & $p<0.001$ & $p=0.97$ & $0.013(0.0077)^{a}$ & $0.023(0.0074)$ \\
\hline $\operatorname{Rec}$ & $p<0.001$ & $p=0.36$ & $p=0.066$ & $0.0068(0.0027)^{a}$ & $p=0.38$ & $p=0.0012$ & $p=0.0010$ & $0.018(0.0073)^{a}$ & $p=0.54$ & $p<0.001$ & $p<0.001$ & $0.036(0.0088)^{a}$ \\
\hline & \multicolumn{4}{|c|}{$F_{(3,21)}=38 ; p<0.001$} & \multicolumn{4}{|c|}{$F_{(3,28)}=14 ; p<0.001$} & \multicolumn{4}{|c|}{$F_{(3,21)}=31 ; p<0.001$} \\
\hline \multicolumn{13}{|c|}{ Parietal cortex-NA } \\
\hline Wake & $0.012(0.0025)^{a}$ & $-0.0021(0.0033)$ & $-0.0049(0.0029)$ & $-0.0032(0.0022)$ & $0.024(0.0071)^{a}$ & $-0.013(0.0058)$ & $-0.012(0.0060)$ & $-0.0068(0.0058)$ & $0.047(0.0088)^{a}$ & $-0.026(0.011)$ & $-0.028(0.010)$ & $-0.0089(0.013)$ \\
\hline Sevo & $p=0.058$ & $0.0099(0.0027)^{a}$ & $-0.0028(0.0034)$ & $-0.0011(0.0029)$ & $p<0.001$ & $0.011(0.0032)^{a}$ & $88 \mathrm{e}-5(0.0062)$ & $0.0061(0.0052)$ & $p<0.001$ & $0.022(0.0075)^{a}$ & $-0.0021(0.0059)$ & $0.017(0.011)$ \\
\hline NA & $p<0.001$ & $p=0.0035$ & $0.0071(0.0023)^{a}$ & $0.0017(0.0012)$ & $p<0.001$ & $p=0.96$ & $0.012(0.0073)^{a}$ & $0.0052(0.0040)$ & $p<0.001$ & $p=0.90$ & $0.020(0.0041)^{a}$ & $0.019(0.0070)$ \\
\hline $\operatorname{Rec}$ & $p<0.001$ & $p=0.54$ & $p=0.16$ & $0.0088(0.0019)^{a}$ & $p<0.001$ & $p=0.0014$ & $p=0.0091$ & $0.017(0.0059)^{a}$ & $p=0.016$ & $p<0.001$ & $p<0.001$ & $0.039(0.0076)^{a}$ \\
\hline & \multicolumn{4}{|c|}{$F_{(3,30)}=12 ; p<0.001$} & \multicolumn{4}{|c|}{$F_{(3,30)}=25 ; p<0.001$} & \multicolumn{4}{|c|}{$F_{(3,30)}=39 ; p<0.001$} \\
\hline
\end{tabular}

${ }^{a}$ Mean ( \pm SD) for each state is shown along the diagonal of each section.

The cells above the mean ( \pm SD) values show the effect sizes as the difference of the means ( \pm SD). Sevo, Sevoflurane administration; NA, NA delivered during sevo; Rec, post-sevoflurane recovery wake.

sevoflurane anesthesia; despite the increase, the temporal complexity in the frontal area (Fig. $6 B$; Table 9) remained significantly lower than that observed during the baseline wake levels $(p<$ 0.001 ), whereas for the parietal area (Fig. $6 D$; Table 9) it was comparable with baseline wake state $(p=0.12)$. The temporal complexity in the frontal and parietal areas during the postsevoflurane recovery wake period remained significantly high compared with sevoflurane anesthesia $(p<0.001)$ (Fig. $6 B, D$; Table 9) but was not significantly different from that observed during baseline wake state $(p \geq 0.2)$ and the NA delivery epoch ( $p \geq 0.089$ ) (Fig. 6B,D; Table 9).
Electroencephalographic spatiotemporal Lempel-Ziv complexity does not correlate with level of consciousness Compared with the baseline wake state, there was a significant decrease $(p<0.001)$ in spatiotemporal complexity during sevoflurane anesthesia (Table 10). Reverse dialysis delivery of $5 \mathrm{~mm}$ carbachol into PFC of these high-density cohort rats (under constant sevoflurane anesthesia) restored wakefulness (defined by attempts or recovery of righting), produced EEG activation, and caused a significant increase in the spatiotemporal complexity $(p<0.001)$ compared with sevoflurane anesthesia; there was no significant difference compared with the baseline wake state $(p=$ 
Table 6. Parietal-to-frontal connectivity before, during, and after sevoflurane administration and NA delivery into prefrontal and parietal cortices

\begin{tabular}{|c|c|c|c|c|c|c|c|c|c|c|c|c|}
\hline & \multicolumn{4}{|l|}{ Low gamma } & \multicolumn{4}{|l|}{ Medium gamma } & \multicolumn{4}{|l|}{ High gamma } \\
\hline & Wake & Sevo & NA & $\operatorname{Rec}$ & Wake & Sevo & NA & $\operatorname{Rec}$ & Wake & Sevo & NA & $\operatorname{Rec}$ \\
\hline \multicolumn{13}{|l|}{ PFC-NA } \\
\hline Wake & $0.010(0.0024)^{a}$ & $-0.0027(0.0020)$ & $-0.0047(0.0026)$ & $-0.0033(0.0019)$ & $0.027(0.010)^{a}$ & $-0.016(0.010)$ & $-0.017(0.012)$ & $-0.0073(0.011)$ & $0.041(0.0088)^{a}$ & $-0.024(0.012)$ & $-0.026(0.013)$ & $-0.0038(0.011)$ \\
\hline Sevo & $p<0.001$ & $0.0077(0.0021)^{a}$ & $-0.0020(0.0011)$ & $-63 e-5(0.0017)$ & $p<0.001$ & $0.011(0.0029)^{a}$ & $-0.0011(0.0022)$ & $0.0085(0.0055)$ & $p<0.001$ & $0.018(0.0078)^{a}$ & $-0.0025(0.0054)$ & $0.020(0.011)$ \\
\hline NA & $p<0.001$ & $p=0.018$ & $0.0058(0.0024)^{a}$ & $0.0013(0.0019)$ & $p<0.001$ & $p=0.99$ & $0.010(0.0036)^{a}$ & $0.0096(0.0063)$ & $p<0.001$ & $p=0.90$ & $0.015(0.0066)^{a}$ & $0.022(0.0082)$ \\
\hline $\operatorname{Rec}$ & $p<0.001$ & $p=0.79$ & $p=0.19$ & $0.0071(0.0026)^{a}$ & $p=0.077$ & $p=0.029$ & $p=0.0094$ & $0.020(0.0049)^{a}$ & $p=0.72$ & $p<0.001$ & $p<0.001$ & $0.038(0.0088)^{a}$ \\
\hline & \multicolumn{4}{|c|}{$F_{(3,21)}=17 ; p<0.001$} & \multicolumn{4}{|c|}{$F_{(3,21)}=13 ; p<0.001$} & \multicolumn{4}{|c|}{$F_{(3,21)}=28 ; p<0.001$} \\
\hline \multicolumn{13}{|c|}{ Parietal cortex-NA } \\
\hline Wake & $0.011(0.0019)^{a}$ & $-0.0014(0.0024)$ & $-0.0029(0.0039)$ & $-0.0024(0.0020)$ & $0.025(0.0066)^{a}$ & $-0.012(0.0047)$ & $-0.012(0.0057)$ & $-0.0051(0.0059)$ & $0.049(0.0086)^{a}$ & $-0.026(0.0095)$ & $-0.029(0.012)$ & $-0.0092(0.014)$ \\
\hline Sevo & $p=0.39$ & $0.0099(0.0021)^{a}$ & $-0.0015(0.0031)$ & $-0.0010(0.0026)$ & $p<0.001$ & $0.013(0.0049)^{a}$ & $69 \mathrm{e}-5(0.0062)$ & $0.0074(0.0054)$ & $p<0.001$ & $0.023(0.0076)^{a}$ & $-0.0026(0.0070)$ & $0.017(0.011)$ \\
\hline NA & $p=0.0060$ & $p=0.33$ & $0.0085(0.0030)^{a}$ & $48 \mathrm{e}-5(0.0032)$ & $p<0.001$ & $p=0.97$ & $0.014(0.0074)^{a}$ & $0.0067(0.0032)$ & $p<0.001$ & $p=0.85$ & $0.020(0.0052)^{a}$ & $0.020(0.0079)$ \\
\hline $\operatorname{Rec}$ & $p=0.032$ & $p=0.66$ & $p=0.95$ & $0.0089(0.0022)^{a}$ & $p=0.0071$ & $p<0.001$ & $p<0.001$ & $0.020(0.0064)^{a}$ & $p=0.019$ & $p<0.001$ & $p<0.001$ & $0.040(0.0083)^{a}$ \\
\hline & \multicolumn{4}{|c|}{$F_{(3,30)}=4.2 ; p=0.014$} & \multicolumn{4}{|c|}{$F_{(3,30)}=28 ; p<0.001$} & \multicolumn{4}{|c|}{$F_{(3,30)}=38 ; p<0.001$} \\
\hline
\end{tabular}

${ }^{a}$ Mean ( \pm SD) for each state is shown along the diagonal of each section.

The cells above the mean ( \pm SD) values show the effect sizes as the difference of the means ( \pm SD). Sevo, Sevoflurane administration; NA, NA delivered during sevo; Rec, post-sevoflurane recovery wake.
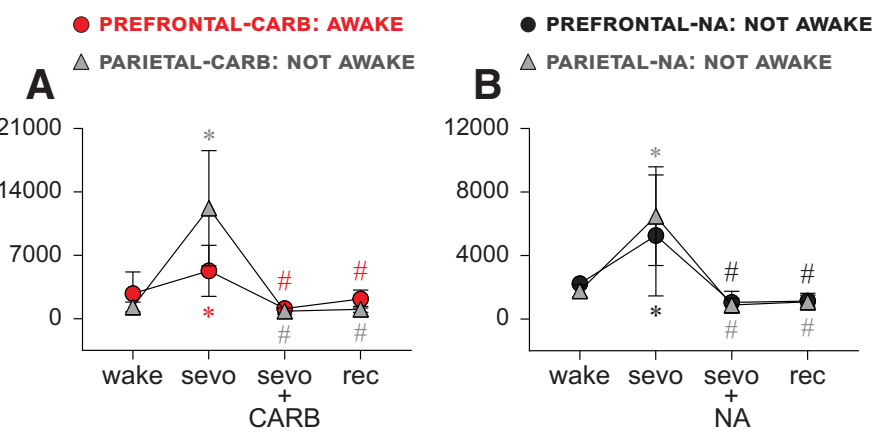

Figure 5. Spectral power of slow oscillations does not correlate with level of consciousness. $A, B$, Spectral power of slow oscillations $(0.5-1 \mathrm{~Hz})$ showed a significant increase during sevoflurane anesthesia (sevo). The restoration of wakefulness through $5 \mathrm{~mm}$ carbachol (CARB) delivery to PFC as well as the absence of wakefulness after CARB delivery to parietal cortex or $20 \mathrm{~mm}$ NA delivery to prefrontal and parietal cortices were reported to be accompanied with EEG activation (Pal et al., 2018), which produced a significant decrease (compared with sevo) in spectral power regardless of the presence or absence of wakefulness $(\boldsymbol{A}, \boldsymbol{B})$. The spectral power during post-sevoflurane recovery wake (rec) epoch remained significantly lower than sevo and was not significantly different from CARB or NA or the baseline wake state (wake) $(\boldsymbol{A}, \boldsymbol{B})$. A linear mixed model with random intercept for each rat was used for the statistical comparisons, and the post hoc pairwise tests were single-step corrected for multiple comparisons. The statistical comparisons are shown at $p<0.05$. The mean, SD, exact $p$ values, F statistics, and the effect sizes for each statistical comparison are provided in Table 7. The significance symbols are color-coded to match the line-symbol plots. *Significant compared with wake. "Significant compared with sevo.

0.58 ) (Table 10). The spatiotemporal complexity during the postsevoflurane recovery wake epoch was significantly higher compared with sevoflurane anesthesia $(p<0.001)$ but was not significantly different from either baseline wake state $(p=0.95)$ or carbachol delivery epoch $(p=0.27)$ (Table 10). These changes in spatiotemporal complexity (Table 10) mirrored the changes in temporal complexity over frontal and parietal areas, as described earlier (Fig. 6A-D). Of note, we also measured changes in local acetylcholine levels before, during, and after sevoflurane anesthesia, as well as carbachol delivery to PFC in these rats. Statistical comparison using linear mixed model showed a significant effect on the acetylcholine levels across conditions $\left(F_{(3,9)}=8.2, p=\right.$ $0.006)$. Compared with the baseline wake state, sevoflurane anesthesia was characterized by an $84 \%$ decrease in acetylcholine levels [mean \pm SD (CI): $0.078 \pm 0.043(0.036-0.12)$ for sevoflurane vs $0.49 \pm 0.35(0.15-0.84)$ for wake state, $p=0.58]$, which is comparable with that reported in our previous publication (Pal et al., 2018). Carbachol delivery to PFC during sevoflurane anesthesia produced a highly significant increase in the local acetylcholine levels [mean \pm SD (CI): $1.57 \pm 0.92(0.66-2.47), p<0.001$ compared with sevoflurane anesthesia], which remained significantly elevated during post-sevoflurane recovery period [mean \pm $\mathrm{SD}(\mathrm{CI}): 1.13 \pm 0.87(0.29-1.99), p=0.007$ compared with sevoflurane]. Overall, these changes in prefrontal acetylcholine levels after sevoflurane administration and carbachol delivery, both in the direction of change and magnitude, are consistent with the data reported in our previous study (Pal et al., 2018).

\section{Discussion}

The divergent effects of cholinergic stimulation on the prefrontal and parietal cortices enabled the discovery that the level of consciousness can be dissociated from EEG measures of cortical functional connectivity and cortical dynamics (Table 11). A number of major theories of consciousness are grounded in the requirement for functional, directed, or effective connectivity across the cortex in the conscious state (Alkire et al., 2008; Dehaene and Changeux, 2011; Baars et al., 2013; Tononi et al., 2016). Indeed, these theories have made successful predictions regarding the reduction in connectivity strength or repertoire empirically observed during sleep, general anesthesia induced by distinct drugs, and pathological states of unconsciousness (Laureys et al., 1999; Massimini et al., 2005; Lee et al., 2009, 2013; Ferrarelli et al., 2010; Boly et al., 2011, 2012; Cimenser et al., 2011; Ku et al., 2011; Changeux, 2012; Lewis et al., 2012; Rosanova et al., 2012; Casali et al., 2013; Jordan et al., 2013; Sarasso et al., 2015; Hudetz and Mashour, 2016; Ranft et al., 2016; Schroeder et al., 2016; Li et al., 2017; Bodart et al., 2018; Sanders et al., 2018a; Uhrig et al., 2018; Hemmings et al., 2019). However, it has been unclear whether the reduction of functional or cortical connectivity played a causal role in the state transition, was associated more closely with loss of higher cognition rather than consciousness per se, or was an epiphenomenon. By dissociating level of consciousness from large-scale cortical connectivity, our findings prompt a reevaluation of the role of these connectivity measures in distinguishing states of consciousness and unconsciousness. Similarly, strategies for clinical monitoring of consciousness based on connectivity patterns (Ferrarelli et al., 2010; Ku et al., 2011; Rosanova et al., 2012; Jordan et al., 2013; Lee et al., 2013) should be reconsidered. Importantly, our findings apply only to the level of consciousness, which can be defined by objective observations, such as wakeful behavior. It is possible 
Table 7. Absolute power of slow oscillations before, during, and after sevoflurane administration, and carbachol and NA delivery into prefrontal and parietal cortices

\begin{tabular}{|c|c|c|c|c|c|c|c|c|c|}
\hline \multicolumn{5}{|c|}{ PFC-carbachol } & \multicolumn{5}{|c|}{ PFC-NA } \\
\hline & Wake & Sevo & CARB & $\operatorname{Rec}$ & & Wake & Sevo & NA & $\operatorname{Rec}$ \\
\hline $\begin{array}{l}\text { Wake } \\
\text { Sevo } \\
\text { CARB } \\
\text { Rec } \\
\text { Parieta }\end{array}$ & $\begin{array}{l}2785(2376)^{a} \\
p=0.021 \\
p=0.23 \\
p=0.90 \\
\end{array}$ & $\begin{array}{l}2506(4309) \\
5291(2824)^{a} \\
p<0.001 \\
p=0.0018 \\
\quad F_{(3,32)}\end{array}$ & $\begin{array}{c}-1654(2476) \\
-4160(3074) \\
1131(722)^{a} \\
p=0.62 \\
3 ; p<0.001\end{array}$ & $\begin{array}{r}-608(2557) \\
-3113(3576) \\
1047(1285) \\
2178(986)^{a}\end{array}$ & $\begin{array}{l}\text { Wake } \\
\text { Sevo } \\
\text { NA } \\
\text { Rec }\end{array}$ & $\begin{array}{l}2225(419)^{a} \\
p=0.0051 \\
p=0.58 \\
p=0.63\end{array}$ & $\begin{array}{l}3040(3746) \\
5265(3811)^{a} \\
p<0.001 \\
p<0.001 \\
\quad F_{(3,3)}=\end{array}$ & $\begin{aligned}-1171(637) \\
-4211(3549) \\
1054(692)^{a} \\
p=1 \\
; p=0.069\end{aligned}$ & $\begin{array}{c}-1087(470) \\
-4127(3519) \\
84(695) \\
1138(371)^{a}\end{array}$ \\
\hline & Wake & Sevo & CARB & $\operatorname{Rec}$ & & Wake & Sevo & NA & $\operatorname{Rec}$ \\
\hline $\begin{array}{l}\text { Wake } \\
\text { Sevo } \\
\text { CARB } \\
\text { Rec }\end{array}$ & $\begin{array}{l}1303(533)^{a} \\
p<0.001 \\
p=0.99 \\
p=1\end{array}$ & $\begin{array}{r}10,900(6044) \\
12,203(6362)^{c} \\
p<0.001 \\
p<0.001 \\
F_{(3,72)}\end{array}$ & $\begin{array}{l}-458(585) \\
-11,3586232) \\
845(337)^{a} \\
p=1 \\
; p<0.001\end{array}$ & $\begin{array}{c}-264(393) \\
-11,163(6251) \\
194(493) \\
1040(328)^{a}\end{array}$ & $\begin{array}{l}\text { Wake } \\
\text { Sevo } \\
\text { NA } \\
\text { Rec }\end{array}$ & $\begin{array}{l}1772(381)^{a} \\
p<0.001 \\
p=0.56 \\
p=0.75\end{array}$ & $\begin{array}{l}4708(3273) \\
6480(3110)^{a} \\
p<0.001 \\
p<0.001 \\
\quad F_{(3,44)}\end{array}$ & $\begin{array}{l}-890(604) \\
-5597(3131) \\
883(419)^{a} \\
p=0.99 \\
; p<0.001\end{array}$ & $\begin{array}{c}-684(769) \\
-5392(3134) \\
205(773) \\
1088(530)^{a}\end{array}$ \\
\hline
\end{tabular}

${ }^{a}$ Mean ( \pm SD) for each state is shown along the diagonal of each section.

The cells above the mean ( \pm SD) values show the effect sizes as the difference of the means ( \pm SD). Sevo, Sevoflurane administration; CARB, carbachol delivered during sevo; NA, NA delivered during sevo; Rec, post-sevoflurane recovery wake.

that disruptions of large-scale cortical connectivity critically impair the phenomenological contents of consciousness, conscious access, or cognitive processing (Mashour and Hudetz, 2017). Furthermore, because of our experimental design requiring a closed, air-tight chamber for continuous sevoflurane exposure, we could not test whether the absence of behavioral arousal or wake state after pharmacological manipulation of the cortex constitutes a truly unresponsive condition. Our inferences are based on the observations of spontaneous behaviors and speak only to the spontaneous changes in level of arousal. Our recent studies in human volunteers (Li et al., 2019) and surgical patients (Vlisides et al., 2019) reported dynamic changes in connectivity patterns under deep anesthesia, but these connectivity patterns were not sufficient to reliably distinguish the changes in the level of consciousness. Although supportive of our results in the current study, it is important to note that these recent studies in humans were restricted to bandwidths below low gamma frequency and entailed long periods of a stable and deep anesthetic plane. A previous study in rats demonstrated that the emergence from isoflurane anesthesia was characterized by multistep discreet fluctuations in spectral power as opposed to a discrete binary process or continuous path to recovery (Hudson et al., 2014). Similar studies in rats with long duration of anesthetics can further clarify whether the connectivity patterns, in particular in gamma frequency, also demonstrate a dynamic evolution.

In both clinical and preclinical settings, the presence of increased spectral power of slow oscillations $(0.5-1 \mathrm{~Hz})$ is considered a signature of unconsciousness (Brown et al., 2010; Lewis et al., 2012; Ní Mhuircheartaigh et al., 2013; Purdon et al., 2013; Warnaby et al., 2017), whereas the appearance of a low-
- PREFRONTAL-NA: NOT AWAKE

B PARIETAL-NA: NOT AWAKE
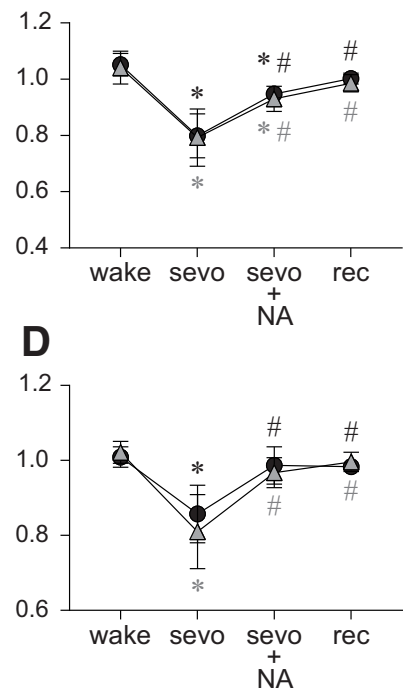

Figure 6. Electroencephalographic temporal Lempel-Ziv complexity does not correlate with level of consciousness. Sevoflurane anesthesia (sevo) was characterized by a significant reduction in Lempel-Ziv complexity in frontal $(\boldsymbol{A}, \boldsymbol{B})$ and parietal areas ( $\boldsymbol{C}$, D). The restoration of wakefulness through $5 \mathrm{~mm}$ carbachol (CARB) delivery to PFC as well as the absence of wakefulness after CARB delivery to parietal cortex or $20 \mathrm{~mm}$ NA delivery to prefrontal and parietal cortices was reported to be accompanied by EEG activation (Pal et al., 2018), which reversed the sevoflurane-induced decrease in complexity. The increase was significant (compared with sevo) for all groups and was not different from baseline wake state (wake), except for prefrontal- and parietal cortex-NA groups $(B-D)$, where it remained significantly lower compared with wake. The complexity during post-sevoflurane recovery wake (rec) epoch remained significantly higher than sevo and was not significantly different from CARB or NA epochs or wake $(\boldsymbol{A}-\boldsymbol{D})$. The plotted values for complexity were obtained after normalizing raw temporal complexity data with phase-randomized surrogate time series and show complexity changes beyond that explained by only spectral changes. A linear mixed model with random intercept for each rat was used for the statistical comparisons, and the post hoc pairwise tests were single-step corrected for multiple comparisons. The statistical comparisons are shown at $p<0.05$. The mean, SD, exact $p$ values, Fstatistics, and the effect sizes for each statistical comparison are provided in Tables 8 and 9. The significance symbols are color-coded to match the line-symbol plots. *Significant compared with baseline wake state. \#Significant compared with sevo.

amplitude fast EEG, along with the dissipation of slow oscillations, correlates with the return of consciousness. In the current study, we did indeed observe the appearance of high-amplitude slow EEG and increase in spectral power of slow oscillations during sevoflurane anesthesia. However, the increased spectral power of slow oscillations was dissipated not only in association with restoration of wakefulness and accompanying EEG activation, an expected finding, but also in the cohorts that showed only 
Table 8. Temporal Lempel-Ziv complexity in frontal and parietal areas before, during, and after sevoflurane administration and carbachol delivery into prefrontal and parietal cortices

\begin{tabular}{|c|c|c|c|c|c|c|c|c|}
\hline & \multicolumn{4}{|l|}{ PFC-carbachol } & \multicolumn{4}{|c|}{ Parietal cortex-carbachol } \\
\hline & Wake & Sevo & CARB & $\operatorname{Rec}$ & Wake & Sevo & CARB & $\operatorname{Rec}$ \\
\hline \multicolumn{9}{|l|}{ Frontal area } \\
\hline Wake & $1.0(0.0390)^{a}$ & $-0.32(0.086)$ & $-0.028(0.11)$ & $-0.055(0.049)$ & $1.0(0.043)^{a}$ & $-0.25(0.092)$ & $-0.062(0.046)$ & $-0.036(0.037)$ \\
\hline Sevo & $p<0.001$ & $0.70(0.092)^{a}$ & $0.29(0.10)$ & $0.27(0.11)$ & $p<0.001$ & $0.78(0.099)^{a}$ & $0.19(0.11)$ & 0.220 .10 \\
\hline CARB & $p=0.80$ & $p<0.001$ & $1.0(0.11)^{a}$ & $-0.027(0.11)$ & $p=0.039$ & $p<0.001$ & $0.97(0.024)^{a}$ & $0.026(0.027)$ \\
\hline $\operatorname{Rec}$ & $p=0.28$ & $p<0.001$ & $p=0.81$ & $0.97(0.028)^{a}$ & $p=0.41$ & $p<0.001$ & $p=0.69$ & $1.0(0.020)^{a}$ \\
\hline & \multirow{2}{*}{\multicolumn{4}{|c|}{$F_{(3,27)}=48 ; p<0.001$}} & \multicolumn{4}{|c|}{$F_{(3,30)}=48 ; p<0.001$} \\
\hline \multicolumn{7}{|l|}{ Parietal area } & & \\
\hline Wake & $1.0(0.035)^{a}$ & $-0.26(0.078)$ & $-0.017(0.060)$ & $-0.026(0.042)$ & $1.0(0.021)^{a}$ & $-0.19(0.077)$ & $-0.017(0.030)$ & $-0.0073(0.026)$ \\
\hline Sevo & $p<0.001$ & $0.75(0.073)^{a}$ & $0.25(0.084)$ & $0.24(0.080)$ & $p<0.001$ & $0.81(0.089)^{a}$ & $0.18(0.095)$ & $0.19(0.089)$ \\
\hline CARB & $p=0.85$ & $p<0.001$ & $1.0(0.050)^{a}$ & $-0.0084(0.057)$ & $p=0.81$ & $p<0.001$ & $0.99(0.019)^{a}$ & $0.0098(0.016)$ \\
\hline $\operatorname{Rec}$ & $p=0.63$ & $p<0.001$ & $p=0.98$ & $0.99(0.022)^{a}$ & $p=0.98$ & $p<0.001$ & $p=0.96$ & $1.0(0.014)^{a}$ \\
\hline & \multicolumn{4}{|c|}{$F_{(3,27)}=66 ; p<0.001$} & \multicolumn{4}{|c|}{$F_{(3,30)}=46 ; p<0.001$} \\
\hline
\end{tabular}

${ }^{a}$ Mean $( \pm S D)$ for each state is shown along the diagonal of each section.

The cells above the mean $( \pm S D$ ) values show the effect sizes as the difference of the means ( \pm SD). Sevo, Sevoflurane administration; CARB, carbachol delivered during sevo; Rec, post-sevoflurane recovery wake.

Table 9. Temporal Lempel-Ziv complexity in frontal and parietal areas before, during, and after sevoflurane administration and NA delivery into prefrontal and parietal cortices

\begin{tabular}{|c|c|c|c|c|c|c|c|c|}
\hline & \multicolumn{4}{|l|}{ PFC-NA } & \multicolumn{4}{|c|}{ Parietal cortex-NA } \\
\hline & Wake & Sevo & NA & $\operatorname{Rec}$ & Wake & Sevo & NA & $\operatorname{Rec}$ \\
\hline \multicolumn{9}{|l|}{ Frontal area } \\
\hline Sevo & $p<0.001$ & $0.80(0.078)^{a}$ & $0.14(0.099)$ & $0.20(0.078)$ & $p<0.001$ & $0.79(0.10)^{a}$ & $0.14(0.097)$ & $0.19(0.12)$ \\
\hline NA & $p<0.001$ & $p<0.001$ & $0.95(0.026)^{a}$ & $0.055(0.038)$ & $p<0.001$ & $p<0.001$ & $0.93(0.045)^{a}$ & $0.055(0.069)$ \\
\hline $\operatorname{Rec}$ & $p=0.15$ & $p<0.001$ & $p=0.089$ & $1.0(0.019)^{a}$ & $p=0.18$ & $p<0.001$ & $p=0.19$ & $0.99(0.031)^{a}$ \\
\hline & \multicolumn{4}{|c|}{$F_{(3,28)}=44 ; p<0.001$} & \multicolumn{4}{|c|}{$F_{(3,40)}=30 ; p<0.001$} \\
\hline Sevo & $p<0.001$ & $0.86(0.077)^{a}$ & $0.13(0.068)$ & $0.13(0.072)$ & $p<0.001$ & $0.81(0.099)^{a}$ & $0.16(0.089)$ & $0.19(0.11)$ \\
\hline NA & $p=0.76$ & $p<0.001$ & $0.99(0.050)^{a}$ & $-0.0035(0.042)$ & $p=0.12$ & $p<0.001$ & $0.97(0.040)^{a}$ & $0.029(0.052)$ \\
\hline \multirow[t]{2}{*}{$\operatorname{Rec}$} & $p=0.67$ & $p<0.001$ & $p=1$ & $0.98(0.011)^{a}$ & $p=0.72$ & $p<0.001$ & $p=0.64$ & $1.0(0.026)^{a}$ \\
\hline & \multicolumn{4}{|c|}{$F_{(3,21)}=19 ; p<0.001$} & \multicolumn{4}{|c|}{$F_{(3,40)}=31 ; p<0.001$} \\
\hline
\end{tabular}

${ }^{a}$ Mean ( \pm SD) for each state is shown along the diagonal of each section.

The cells above the mean ( \pm SD) values show the effect sizes as the difference of the means ( \pm SD). Sevo, Sevoflurane administration; NA, NA delivered during sevo; Rec, post-sevoflurane recovery wake.

Table 10. Spatiotemporal Lempel-Ziv complexity before, during, and after sevoflurane administration and carbachol delivery into PFC

\begin{tabular}{lllll}
\hline & \multicolumn{4}{l}{ Prefrontal-carbachol } \\
\cline { 2 - 5 } & Wake & Sevo & CARB & Rec \\
\hline Wake & $0.92(0.018)^{a}$ & $-0.16(0.024)$ & $-0.017(0.020)$ & $0.0072(0.014)$ \\
Sevo & $p<0.001$ & $0.76(0.033)^{a}$ & $0.14(0.042)$ & $0.17(0.019)$ \\
CARB & $p=0.58$ & $p<0.001$ & $0.90(0.031)^{a}$ & $0.024(0.033)$ \\
Rec & $p=0.95$ & $p<0.001$ & $p=0.27$ & $0.92(0.022)^{a}$ \\
& $F_{(3,9)}=69 ; p<0.001$ & & \\
\hline
\end{tabular}

$a^{a}$ Mean ( \pm SD) for each state is shown along the diagonal of each section.

The cells above the mean ( \pm SD) values show the effect sizes as the difference of the means ( \pm SD). Sevo, Sevoflurane administration; CARB, carbachol delivered during sevo; Rec, post-sevoflurane recovery wake.

EEG activation without any signs of wakefulness. These findings demonstrate that dissipation of slow oscillations correlates with an activated EEG rather than behavioral arousal, per se. Furthermore, the known occurrence of low-amplitude fast EEG, a pattern typically associated with wakefulness, during rapid eye movement sleep (Hobson, 2009), the occurrence of highamplitude slow-wave EEG during wakefulness in patients with Angelman syndrome (Sidorov et al., 2017), and the experimental induction of high-amplitude slow-wave EEG in freely moving rats through systemic atropine administration (Qiu et al., 2015) all show various instances of dissociation between EEG patterns and level of consciousness. Similar reports from human subjects under general anesthesia further support these findings across spe-
Table 11. Summary of changes in cortical dynamics in the presence and absence of wakefulness during continuous sevoflurane administration and pharmacological manipulations

\begin{tabular}{lll}
\hline & Wakefulness $^{a}$ & No signs of wakefulness \\
\hline Cortical functional connectivity & Low & Low \\
Cortical slow oscillation power & Low & Low \\
Cortical complexity & High & High \\
\hline
\end{tabular}

${ }^{a}$ Carbachol delivery to PFC.

${ }^{b}$ Carbachol delivery to parietal, or NA delivery to prefrontal and parietal, cortices.

cies and states of unconsciousness. For instance, using the isolated forearm technique, Gaskell et al. (2017) demonstrated that the presence of frontal $\alpha-\delta$ pattern in the EEG, typically considered to be a marker of anesthetic-induced unconsciousness, cannot reliably discriminate between behavioral responsiveness and unresponsiveness in certain patients under anesthesia, a conclusion that was supported by another patient report (Sanders et al., 2018b). Conversely, ketamine is known to produce fast EEG signatures that, unlike other commonly used general anesthetics, such as propofol or sevoflurane, are not reliably tracked by anesthesia monitors (Maksimow et al., 2006). Our data are consistent with these past findings by demonstrating that slow oscillations can be dissociated from level of consciousness.

Electroencephalographic complexity has been demonstrated to be high during wakefulness as well as in states with high phenomenological contents, such as rapid eye movement sleep (Abá- 
solo et al., 2015; Schartner et al., 2017b) and the psychedelic experience (Tagliazucchi et al., 2014; Schartner et al., 2017a; Li and Mashour, 2019). On the other hand, complexity has been reported to be low during the states with reduced level of arousal, such as non-rapid eye movement sleep (Casali et al., 2013; Abásolo et al., 2015; Schartner et al., 2017b) and anesthesia (Casali et al., 2013; Hudetz et al., 2015, 2016; Sarasso et al., 2015). Based on these earlier reports, we expected that EEG complexity would increase after restoration of wakefulness, as achieved by prefrontal cholinergic stimulation, while remaining at a reduced level in the cohorts that failed to demonstrate restoration of wakefulness. Contrary to our expectation, temporal complexity, as measured through Lempel-Ziv algorithm, increased regardless of the presence or absence of wakefulness and instead correlated with EEG activation. In our earlier study (Pal et al., 2018), we showed that the restoration of wakefulness following carbachol-mediated cholinergic stimulation of PFC was accompanied by an approximately fivefold increase in local acetylcholine levels. More limited increases in local acetylcholine levels were also observed after NA delivery to PFC as well as carbachol or NA delivery to parietal cortex. In the current study, the changes in spatiotemporal Lempel-Ziv complexity also showed a positive relationship with prefrontal cholinergic tone and EEG activation, which aligns with the changes observed in temporal Lempel-Ziv complexity. Furthermore, the increase in complexity has been reported in states, such as rapid eye movement sleep and ketamine, which are characterized by increased cholinergic tone and EEG activation (Lydic and Baghdoyan, 2005; Abásolo et al., 2015; Pal et al., 2015; Sarasso et al., 2015; Schartner et al., 2017a). Therefore, it is possible that underlying changes in cholinergic tone are more closely associated with the complexity changes rather than behavioral arousal.

Interestingly, despite the increase in cortical acetylcholine levels or the behavioral phenotype following carbachol or NA delivery into prefrontal and parietal cortices (Pal et al., 2018), the functional connectivity, as measured in the current study, did not show a significant change. Therefore, functional connectivity is doubly dissociated from both behavior and cortical cholinergic tone. Furthermore, our results are consistent with the effect of anesthetics on local neuronal networks. A dissociation between cortical neuronal interactions and spontaneous behavior was demonstrated after stimulation of the ascending arousal system in rats anesthetized with desflurane at comparable effective levels to that used here (Pillay et al., 2014). Notably, previous studies targeting multiple subcortical pathways with a variety of stimulus modalities (electrical, pharmacological, optogenetic) have demonstrated restoration of wakefulness from unconsciousness induced by different classes of anesthetics (Alkire et al., 2007, 2009; Solt et al., 2011, 2014; Chemali et al., 2012; Muindi et al., 2016; Taylor et al., 2016; Gao et al., 2019), suggesting that site of stimulation, rather than stimulus modality or anesthetic agent used for inducing unconsciousness, may play a greater role in modulating behavioral arousal. However, these studies did not investigate changes in connectivity or complexity; therefore, it remains to be seen whether the restoration of wakefulness from anesthesia after subcortical stimulation will produce connectivity and complexity patterns similar to those observed in our study.

Although previous reports of the dissociation between EEG and behavioral states (Maksimow et al., 2006; Hobson, 2009; Qiu et al., 2015; Gaskell et al., 2017; Sidorov et al., 2017; Sanders et al., 2018b) are supportive of our results, caution should be applied in the interpretation of these findings because of the reliance of our study on behavioral motor activity for the determination of wakefulness, the inherent limitations of the connectivity and complexity measures used, and the inability to make any inferences related to the content or experiential nature of conscious activity. Furthermore, although we have artificially dissociated levels of consciousness and various cortical measures, it is still possible that they correlate during more typical physiological and pharmacological state transitions or with other techniques of neurophysiology, functional neuroimaging, and analysis.

In conclusion, we conducted a systematic comparison of multiple EEG measures (connectivity, complexity, and spectral power) after cholinergic or noradrenergic stimulation of distinct cortical areas, and demonstrate the dissociation between these cortical measures and level of consciousness.

\section{References}

Abásolo D, Simons S, Morgado da Silva R, Tononi G, Vyazovskiy VV (2015) Lempel-Ziv complexity of cortical activity during sleep and waking in rats. J Neurophysiol 113:2742-2752.

Alkire MT, McReynolds JR, Hahn EL, Trivedi AN (2007) Thalamic microinjection of nicotine reverses sevoflurane-induced loss of righting reflex in the rat. Anesthesiology 107:264-272.

Alkire MT, Hudetz AG, Tononi G (2008) Consciousness and anesthesia. Science 322:876-880.

Alkire MT, Asher CD, Franciscus AM, Hahn EL (2009) Thalamic microinfusion of antibody to a voltage-gated potassium channel restores consciousness during anesthesia. Anesthesiology 110:766-773.

Baars BJ, Franklin S, Ramsoy TZ (2013) Global workspace dynamics: cortical "binding and propagation" enables conscious contents. Front Psychol $4: 200$.

Bodart O, Amico E, Gómez F, Casali AG, Wannez S, Heine L, Thibaut A, Annen J, Boly M, Casarotto S, Rosanova M, Massimini M, Laureys S, Gosseries O (2018) Global structural integrity and effective connectivity in patients with disorders of consciousness. Brain Stimul 11:358-365.

Boly M, Garrido MI, Gosseries O, Bruno MA, Boveroux P, Schnakers C, Massimini M, Litvak V, Laureys S, Friston K (2011) Preserved feedforward but impaired top-down processes in the vegetative state. Science 332:858-862.

Boly M, Moran R, Murphy M, Boveroux P, Bruno MA, Noirhomme Q, Ledoux D, Bonhomme V, Brichant JF, Tononi G, Laureys S, Friston K (2012) Connectivity changes underlying spectral EEG changes during propofol-induced loss of consciousness. J Neurosci 32:7082-7090.

Bonhomme V, Vanhaudenhuyse A, Demertzi A, Bruno MA, Jaquet O, Bahri MA, Plenevaux A, Boly M, Boveroux P, Soddu A, Brichant JF, Maquet P, Laureys S (2016) Resting-state network-specific breakdown of functional connectivity during ketamine alteration of consciousness in volunteers: Anesthesiology 125:873-888.

Borjigin J, Lee U, Liu T, Pal D, Huff S, Klarr D, Sloboda J, Hernandez J, Wang MM, Mashour GA (2013) Surge of neurophysiological coherence and connectivity in the dying brain. Proc Natl Acad Sci U S A 110:1443214437.

Brown EN, Lydic R, Schiff ND (2010) General anesthesia, sleep, and coma. N Engl J Med 363:2638-2650.

Carhart-Harris RL (2018) The entropic brain: revisited. Neuropharmacology 142:167-178.

Casali AG, Gosseries O, Rosanova M, Boly M, Sarasso S, Casali KR, Casarotto S, Bruno MA, Laureys S, Tononi G, Massimini M (2013) A theoretically based index of consciousness independent of sensory processing and behavior. Sci Transl Med 5:198ra105.

Castro S, Falconi A, Chase MH, Torterolo P (2013) Coherent neocortical $40-\mathrm{Hz}$ oscillations are not present during REM sleep. Eur J Neurosci 37:1330-1339.

Castro S, Cavelli M, Vollono P, Chase MH, Falconi A, Torterolo P (2014) Inter-hemispheric coherence of neocortical gamma oscillations during sleep and wakefulness. Neurosci Lett 578:197-202.

Changeux JP (2012) Conscious processing: implications for general anesthesia. Curr Opin Anaesthesiol 25:397-404.

Chemali JJ, Van Dort CJ, Brown EN, Solt K (2012) Active emergence from propofol general anesthesia is induced by methylphenidate. Anesthesiology 116:998-1005. 
Cimenser A, Purdon PL, Pierce ET, Walsh JL, Salazar-Gomez AF, Harrell PG, Tavares-Stoeckel C, Habeeb K, Brown EN (2011) Tracking brain states under general anesthesia by using global coherence analysis. Proc Natl Acad Sci U S A 108:8832-8837.

Dehaene S, Changeux JP (2011) Experimental and theoretical approaches to conscious processing. Neuron 70:200-227.

Delorme A, Makeig S (2004) EEGLAB: an open source toolbox for analysis of single-trial EEG dynamics including independent component analysis. J Neurosci Methods 134:9-21.

Ferrarelli F, Massimini M, Sarasso S, Casali A, Riedner BA, Angelini G, Tononi G, Pearce RA (2010) Breakdown in cortical effective connectivity during midazolam-induced loss of consciousness. Proc Natl Acad Sci U S A 107:2681-2686.

Gao S, Proekt A, Renier N, Calderon DP, Pfaff DW (2019) Activating an anterior nucleus gigantocellularis subpopulation triggers emergence from pharmacologically-induced coma in rodents. Nat Commun 10:2897.

Gaskell AL, Hight DF, Winders J, Tran G, Defresne A, Bonhomme V, Raz A, Sleigh JW, Sanders RD (2017) Frontal alpha- $\delta$ EEG does not preclude volitional response during anaesthesia: prospective cohort study of the isolated forearm technique. Br J Anaesth 119:664-673.

Hemmings HC Jr, Riegelhaupt PM, Kelz MB, Solt K, Eckenhoff RG, Orser BA, Goldstein PA (2019) Towards a comprehensive understanding of anesthetic mechanisms of action: a decade of discovery. Trends Pharmacol Sci 40:464-481.

Hobson JA (2009) REM sleep and dreaming: towards a theory of protoconsciousness. Nat Rev Neurosci 10:803-813.

Hudetz AG (2012) General anesthesia and human brain connectivity. Brain Connect 2:291-302.

Hudetz AG, Mashour GA (2016) Disconnecting consciousness: is there a common anesthetic end point? Anesth Analg 123:1228-1240.

Hudetz AG, Liu X, Pillay S (2015) Dynamic repertoire of intrinsic brain states is reduced in propofol-induced unconsciousness. Brain Connect $5: 10-22$.

Hudetz AG, Liu X, Pillay S, Boly M, Tononi G (2016) Propofol anesthesia reduces Lempel-Ziv complexity of spontaneous brain activity in rats. Neurosci Lett 628:132-135.

Hudson AE, Calderon DP, Pfaff DW, Proekt A (2014) Recovery of consciousness is mediated by a network of discrete metastable activity states. Proc Natl Acad Sci U S A 111:9283-9288.

Jordan D, Ilg R, Riedl V, Schorer A, Grimberg S, Neufang S, Omerovic A, Berger S, Untergehrer G, Preibisch C, Schulz E, Schuster T, Schröter M, Spoormaker V, Zimmer C, Hemmer B, Wohlschläger A, Kochs EF, Schneider G (2013) Simultaneous electroencephalographic and functional magnetic resonance imaging indicate impaired cortical top-down processing in association with anesthetic-induced unconsciousness: Anesthesiology 119:1031-1042.

Kenny JD, Taylor NE, Brown EN, Solt K (2015) Dextroamphetamine (but not atomoxetine) induces reanimation from general anesthesia: implications for the roles of dopamine and norepinephrine in active emergence. PLoS One 10:e131914.

Ku SW, Lee U, Noh GJ, Jun IG, Mashour GA (2011) Preferential inhibition of frontal-to-parietal feedback connectivity is a neurophysiologic correlate of general anesthesia in surgical patients. PLoS One 6:e25155.

Laureys S, Goldman S, Phillips C, Van Bogaert P, Aerts J, Luxen A, Franck G, Maquet P (1999) Impaired effective cortical connectivity in vegetative state: preliminary investigation using PET. Neuroimage 9:377-382.

Lee U, Kim S, Noh GJ, Choi BM, Hwang E, Mashour GA (2009) The directionality and functional organization of frontoparietal connectivity during consciousness and anesthesia in humans. Conscious Cogn 18:10691078.

Lee U, Ku S, Noh G, Baek S, Choi B, Mashour GA (2013) Disruption of frontal-parietal communication by ketamine, propofol, and sevoflurane. Anesthesiology 118:1264-1275.

Lempel A, Ziv J (1976) On the complexity of finite sequences. IEEE Trans Inf Theory 22:75-81.

Lewis LD, Weiner VS, Mukamel EA, Donoghue JA, Eskandar EN, Madsen JR, Anderson WS, Hochberg LR, Cash SS, Brown EN, Purdon PL (2012) Rapid fragmentation of neuronal networks at the onset of propofolinduced unconsciousness. Proc Natl Acad Sci U S A 109:E3377-E3386.

Li D, Mashour GA (2019) Cortical dynamics during psychedelic and anesthetized states induced by ketamine. Neuroimage 196:32-40.
Li D, Hambrecht-Wiedbusch VS, Mashour GA (2017) Accelerated recovery of consciousness after general anesthesia is associated with increased functional brain connectivity in the high-gamma bandwidth. Front Syst Neurosci 11:16

Li D, Vlisides PE, Kelz MB, Avidan MS, Mashour GA (2019) Dynamic cortical connectivity during general anesthesia in healthy volunteers. Anesthesiology 130:870-884.

Lydic R, Baghdoyan HA (2005) Sleep, anesthesiology, and the neurobiology of arousal state control. Anesthesiology 103:1268-1295.

Maksimow A, Särkelä M, Långsjö JW, Salmi E, Kaisti KK, Yli-Hankala A, Hinkka-Yli-Salomäki S, Scheinin H, Jääskeläinen SK (2006) Increase in high frequency EEG activity explains the poor performance of EEG spectral entropy monitor during S-ketamine anesthesia. Clin Neurophysiol 117:1660-1668

Mashour GA, Hudetz AG (2017) Bottom-up and top-down mechanisms of general anesthetics modulate different dimensions of consciousness. Front Neural Circuits 11:44.

Massimini M, Ferrarelli F, Huber R, Esser SK, Singh H, Tononi G (2005) Breakdown of cortical effective connectivity during sleep. Science 309:2228-2232.

Muindi F, Kenny JD, Taylor NE, Solt K, Wilson MA, Brown EN, Van Dort CJ (2016) Electrical stimulation of the parabrachial nucleus induces reanimation from isoflurane general anesthesia. Behav Brain Res 306:20-25.

Ní Mhuircheartaigh R, Warnaby C, Rogers R, Jbabdi S, Tracey I (2013) Slow-wave activity saturation and thalamocortical isolation during propofol anesthesia in humans. Sci Transl Med 5:208ra148.

Pal D, Hambrecht-Wiedbusch VS, Silverstein BH, Mashour GA (2015) Electroencephalographic coherence and cortical acetylcholine during ketamine-induced unconsciousness. Br J Anaesth 114:979-989.

Pal D, Silverstein BH, Lee H, Mashour GA (2016) Neural correlates of wakefulness, sleep, and general anesthesia: an experimental study in rat. Anesthesiology 125:929-942.

Pal D, Dean JG, Liu T, Li D, Watson CJ, Hudetz AG, Mashour GA (2018) Differential role of prefrontal and parietal cortices in controlling level of consciousness. Curr Biol 28:2145-2152.e5.

Paxinos C, Watson G (2007) The rat brain in stereotaxic coordinates, Ed 6 . London: Academic.

Pillay S, Vizuete J, Liu X, Juhasz G, Hudetz AG (2014) Brainstem stimulation augments information integration in the cerebral cortex of desflurane-anesthetized rats. Front Integr Neurosci 8:8.

Purdon PL, Pierce ET, Mukamel EA, Prerau MJ, Walsh JL, Wong KF, SalazarGomez AF, Harrell PG, Sampson AL, Cimenser A, Ching S, Kopell NJ, Tavares-Stoeckel C, Habeeb K, Merhar R, Brown EN (2013) Electroencephalogram signatures of loss and recovery of consciousness from propofol. Proc Natl Acad Sci U S A 110:E1142-E1151.

Qiu MH, Chen MC, Lu J (2015) Cortical neuronal activity does not regulate sleep homeostasis. Neuroscience 297:211-218.

R Core Team (2019) R: a language and environment for statistical computing. R Foundation for Statistical Computing. https://www.R-project.org/.

Ranft A, Golkowski D, Kiel T, Riedl V, Kohl P, Rohrer G, Pientka J, Berger S, Thul A, Maurer M, Preibisch C, Zimmer C, Mashour GA, Kochs EF, Jordan D, Ilg R (2016) Neural correlates of sevoflurane-induced unconsciousness identified by simultaneous functional magnetic resonance imaging and electroencephalography: Anesthesiology 125:861-872.

Rosanova M, Gosseries O, Casarotto S, Boly M, Casali AG, Bruno MA, Mariotti M, Boveroux P, Tononi G, Laureys S, Massimini M (2012) Recovery of cortical effective connectivity and recovery of consciousness in vegetative patients. Brain 135:1308-1320.

Sanders RD, Banks MI, Darracq M, Moran R, Sleigh J, Gosseries O, Bonhomme V, Brichant JF, Rosanova M, Raz A, Tononi G, Massimini M, Laureys S, Boly M (2018a) Propofol-induced unresponsiveness is associated with impaired feedforward connectivity in cortical hierarchy. Br J Anaesth 121:1084-1096.

Sanders RD, Mostert N, Lindroth H, Tononi G, Sleigh J (2018b) Is consciousness frontal? Two perioperative case reports that challenge that concept. Br J Anaesth 121:330-332.

Sarasso S, Boly M, Napolitani M, Gosseries O, Charland-Verville V, Casarotto S, Rosanova M, Casali AG, Brichant JF, Boveroux P, Rex S, Tononi G, Laureys S, Massimini M (2015) Consciousness and complexity during unresponsiveness induced by propofol, xenon, and ketamine. Curr Biol 25:3099-3105. 
Schartner MM, Seth A, Noirhomme Q, Boly M, Bruno MA, Laureys S, Barrett A (2015) Complexity of multi-dimensional spontaneous EEG decreases during propofol induced general anaesthesia. PLoS One 10:e0133532.

Schartner MM, Carhart-Harris RL, Barrett AB, Seth AK, Muthukumaraswamy SD (2017a) Increased spontaneous MEG signal diversity for psychoactive doses of ketamine, LSD and psilocybin. Sci Rep 7:46421.

Schartner MM, Pigorini A, Gibbs SA, Arnulfo G, Sarasso S, Barnett L, Nobili L, Massimini M, Seth AK, Barrett AB (2017b) Global and local complexity of intracranial EEG decreases during NREM sleep. Neurosci Conscious 2017:niw022.

Schroeder KE, Irwin ZT, Gaidica M, Nicole Bentley J, Patil PG, Mashour GA, Chestek CA (2016) Disruption of corticocortical information transfer during ketamine anesthesia in the primate brain. Neuroimage 134:459465.

Schrouff J, Perlbarg V, Boly M, Marrelec G, Boveroux P, Vanhaudenhuyse A, Bruno MA, Laureys S, Phillips C, Pélégrini-Issac M, Maquet P, Benali H (2011) Brain functional integration decreases during propofol-induced loss of consciousness. Neuroimage 57:198-205.

Sidorov MS, Deck GM, Dolatshahi M, Thibert RL, Bird LM, Chu CJ, Philpot BD (2017) Delta rhythmicity is a reliable EEG biomarker in Angelman syndrome: a parallel mouse and human analysis. J Neurodev Disord 9:17.

Solt K, Cotten JF, Cimenser A, Wong KF, Chemali JJ, Brown EN (2011) Methylphenidate actively induces emergence from general anesthesia. Anesthesiology 115:791-803.

Solt K, Van Dort CJ, Chemali JJ, Taylor NE, Kenny JD, Brown EN (2014)
Electrical stimulation of the ventral tegmental area induces reanimation from general anesthesia. Anesthesiology 121:311-319.

Tagliazucchi E, Carhart-Harris R, Leech R, Nutt D, Chialvo DR (2014) Enhanced repertoire of brain dynamical states during the psychedelic experience: enhanced repertoire of brain dynamical states. Hum Brain Mapp 35:5442-5456.

Taylor NE, Van Dort CJ, Kenny JD, Pei J, Guidera JA, Vlasov KY, Lee JT, Boyden ES, Brown EN, Solt K (2016) Optogenetic activation of dopamine neurons in the ventral tegmental area induces reanimation from general anesthesia. Proc Natl Acad Sci U S A 113:12826-12831.

Tononi G, Boly M, Massimini M, Koch C (2016) Integrated information theory: from consciousness to its physical substrate. Nat Rev Neurosci $17: 450-461$.

Uhrig L, Sitt JD, Jacob A, Tasserie J, Barttfeld P, Dupont M, Dehaene S, Jarraya B (2018) Resting-state dynamics as a cortical signature of anesthesia in monkeys. Anesthesiology 129:942-958.

Vlisides PE, Li D, Zierau M, Lapointe AP, Ip KI, McKinney AM, Mashour GA (2019) Dynamic cortical connectivity during general anesthesia in surgical patients. Anesthesiology 130:885-897.

Warnaby CE, Sleigh JW, Hight D, Jbabdi S, Tracey I (2017) Investigation of slow-wave activity saturation during surgical anesthesia reveals a signature of neural inertia in humans. Anesthesiology 127:645-657.

Ziv J, Lempel A (1977) A universal algorithm for sequential data compression. IEEE Trans Inf Theory 23:337-343.

Ziv J, Lempel A (1978) Compression of individual sequences via variablerate coding. IEEE Trans Inf Theory 24:530-536. 